\title{
The AGS Ggamma meter and calibrating the gauss clock
}

\author{
L. Ahrens
}

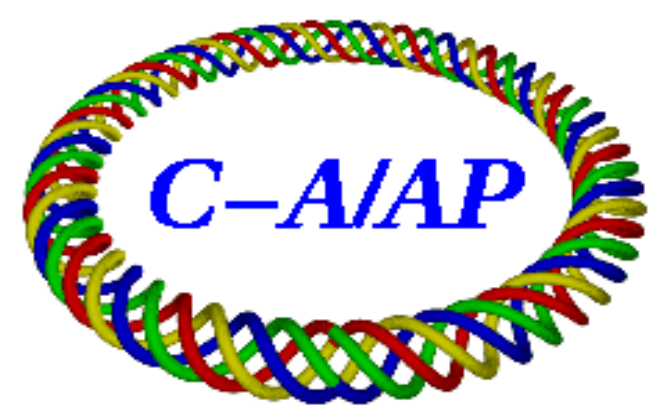

\section{Collider-Accelerator Department Brookhaven National Laboratory \\ Upton, NY 11973}

Notice: This document has been authorized by employees of Brookhaven Science Associates, LLC under Contract No. DE-AC02-98CH10886 with the U.S. Department of Energy. The United States Government retains a nonexclusive, paid-up, irrevocable, world-wide license to publish or reproduce the published form of this document, or allow others to do so, for United States Government purposes. 
The AGS Ggamma Meter and Calibrating the Gauss Clock

Leif Ahrens March, 2014

Contents:

I. Background, motivation, and a first look at beam data $p 2$

II. Model description and a suggested parameter optimization strategy $p 6$

III. Real data study

A. The machinery - the logged data and spreadsheet analysis being used. One data set analysis in detail. $p 9$

B. 2013: Some example sets and parameters summary plots $p 17$

C. 2012: An experimentally poor JQ timing set generation, associated JQ on and off data, fitting issues $p 21$

IV. A List of Action Items $p 24$

V. Appendix 1: Error Analysis and the Ggamma measurements at Low Energies p25

VI. Appendix 2: Correcting the measured frequency for $\mathbf{d}^{\mathbf{2}} \mathbf{F} / \mathbf{d t}^{2}$ p 28

VII. Power Point Presentation at Haixin's Polarization meeting 12Feb14 p30 


\section{Background, motivation, and a first look at beam data}

During AGS Polarized Proton acceleration periods, one output from the AGS Ggamma Meter, namely the energy (or Ggamma) calculated from the magnetic field in the AGS main magnets and the beam radius - both measured at a particular instant, is used to figure out the times in the AGS magnet acceleration cycle when the beam passes through a particular set of depolarizing resonances. The resonance condition set occur whenever a particle's Ggamma (or energy *(G/m), with $\mathrm{G}$ and $\mathrm{m}$ constants) becomes nearly equal to $n^{*} Q x$ (i.e. any integer multiplied by the horizontal betatron tune). If we know Ggamma(t) we can figure out when in time each resonance is crossed. Because of this deliverable, this machinery is referred to as the "Ggamma Meter" rather than the AGS energy meter - just words.

For the AGS Tune Jump system, the resonance crossing times must be known with excellent accuracy. Pulsing the quadrupoles of the Tune Jump system will speed the beam across the resonance condition provided the timing is close enough. What are the Jump Quad system parameters? Consider a single particle accelerating in the AGS, a horizontal tune jump of 0.04 , a quad-jumping time interval of $100 \mathrm{usec}$, and a beam acceleration rate of 50 units of Ggamma in $500 \mathrm{~ms}$, (or 0.025 units of Ggamma in $250 \mathrm{usec})$. For these numbers - which are close to the numbers for the existing system - it follows that the timing of the tune jumping must occur within $+/-250$ usec of the perfect jump time for this particle or else the particle will cross the resonance with no beneficial speeding up. The beam energy (in units of Ggamma) changes by $\mathbf{0 . 0 2 5}$ in 250 usec. If our energy at a given time prediction is off by 0.025 (Ggamma units) the Tune Jump system will not improve the polarization. How much better than this the timing needs to be for optimal correction depends on the beam energy and the tune spread. Adding a tune spread to the beam reduces the allowed window for minimal depolarization loss but widens the window that provides some benefit from the jump.

We choose to control when the tune jumping occurs via real time - measured from AGS T0. The resonance crossing times are learned from a series of measurements of (beam energy and horizontal tune) taken at approximately the times when the resonances are crossed. This beam energy or Ggamma or here GgammaBr - the nomenclature gets picky - comes (via the Ggamma Meter) from measuring the AGS magnetic field using the gauss meter and measuring the beam momentum offset using the $\mathrm{r}$, the radial average from the beam position measuring system. The starting field for the gauss clock (gauss at T0) and the calibration for the gauss clock (GCcal = gauss clock counts/gauss) need to be determined. This is accomplished by requiring these estimates of beam energy using magnetic field and radius - GgammaBr - equal a second very different set of energy estimates. This second set uses the beam frequency $(F)$ and beam path length (meaning the design path length $2 \pi R 0$ but with R 0 experimentally shifted by the same $\mathrm{r}$ mentioned above) and is designated GgammaFr. In the short-hand used here, the Ggamma Meter provides the two energy estimates at each time - GgammaBr and GgammaFr. The calibration parameters are chosen to made the two sets as equal as possible throughout the acceleration cycle; adjusting B0, GCcal, and R0. 
The Ggamma Meter takes as inputs a set of measurements of frequency $(\mathrm{F}(\mathrm{t}))$, radius $(\mathrm{r}(\mathrm{t}))$, and gauss clock counts $(\mathrm{GCC}(\mathrm{t}))$. The frequency is a measure of the bunch or $r f$ frequency. The AGS orbit display application calculates $r(t)$ as the average of the measured positions at all the horizontal pickups. The pick up locations are dead reckoned to give $r=0$ if the beam is at the design radius, $R 0$. The beam path length is then taken to be $2 \pi(\mathrm{R} 0+\mathrm{r})$ and the beam velocity and energy follow from this path length and the revolution frequency $(\mathrm{F} / \mathrm{h})$ with $\mathrm{h}$ the rf cavity acceleration harmonic. This gives GgammaFr(t). The other energy (GgammaBr) assumes the field when the gauss clock starts counting is known. The change in field to time $t$ is given by the measured accumulated gauss clock counts multiplied by the gauss clock calibration (gauss/GCC). In order to deal with experimental data, this calibration factor gets an added ad hoc complication, namely a correction dependent on the rate of change the counting rate. The Ggamma meter takes GCC $(t)$ and together with the past history for this cycle calculates $\mathrm{B}(\mathrm{t})$. From this the momentum on the central radius can be calculated (given the AGS radius of curvature), and with $r$ a correction applied to learn the momentum shift to the measured radius.

To summarize and review: the Ggamma Meter evaluates two functions of time at whatever times we choose to provide the input measurements.

The Ggamma Meter calculated GgammaFr(t) from $F(t), r(t)$ and the constant R0.

Function \#1: GgammaFr $=G^{*} \gamma, \gamma=1 / \sqrt{ }\left(1-\beta^{2}\right), \beta=(1 / c) *\left(\operatorname{Frev}^{*} 2 \pi(\mathrm{R} 0+\mathrm{r})\right.$

The Ggamma Meter calculates GgammaBr(t) from $\operatorname{GCC}(t), r(t)$ and constants GCcal, the basic counts per gauss, B0, the starting field, and - because we will introduce it later on - an additional correction with a proportionality coefficient "Bdd", to allow for a dependence on changes in the slope $\mathrm{dB} / \mathrm{dt}$. The same measured $\mathrm{r}$ is here used to correct the momentum calculated from B (and the radius of curvature on the central orbit) to account for the beam not being on the central orbit. It is assumed that a beam with $r=0$ is on the design orbit with the design rigidity.

Function \#2: GgammaBr $=G^{*} \gamma, \gamma=E / M=\left(\sqrt{ }\left(P^{2}+M^{2}\right)\right) / M, P=P o\left(1+(r / R 0)^{*} \gamma_{t r}{ }^{2}\right), P o=$ $\left.\mathrm{B}^{*} \rho^{*} \mathrm{Q} * \mathrm{c}\right), \mathrm{B}=(\mathrm{B} 0+\mathrm{GCC} * \mathrm{GCcal})$ and $\mathrm{GCcal}$ can be given a dependence on $\mathrm{d} / \mathrm{dt}(\mathrm{dB} / \mathrm{dt})$ if the coefficient Bdd is not zero.

There are more constants in these equations than specifically called out in the above text, (M, the proton mass; $\mathrm{Q}$, the proton charge; $\rho$, the radius of curvature on the design orbit; $\gamma_{\mathrm{tr}}$, transition gamma) but these others are either well known or not critically sensitive players.

The main subject for this note is just understanding how best to learn these calibration coefficients. (The basic arguments - the lack of degeneracy between GgammaFr and GgammaBr, the fitting approach - were made rather crisply by Dr. Howard Weisberg in 
AGS tech note \#145 back in 1978. He was measuring the AGS extraction energy for the slow-extracted AGS beam despite working at a main magnet excitation current that pushed the magnet iron well into saturation and produced extraction momenta one or two percent below an unsaturated machine). One measures GgammaBr(i) and GgammaFr(i) at $\mathrm{n}$ times during the cycle, $(\mathrm{i}=1, \mathrm{n})$. One takes the differences between these pairs of measurements, delta(i) = GgammaBr(i) - GgammaFr(i); weights this deltas by the errors in the differences, and forms the sum of the squares of these weighted differences over the $n$ measurements. The "best" calibration numbers are then obtained by allowing these calibration numbers to vary so as to minimize this sum, usually using a nonlinear leastsquares fitting algorithm. Because of the energy range covered in the AGS - from a beta of 0.9 to beta of greater than 0.99 the two Ggamma functions are not degenerate. The fitting can work.

We next see how this goes for one rather good set of data (23May13, 231 measurements). The difference GgammaFr-GgammaBr is plotted vs time in the cycle in figure 1. For the left plot initial calibration values are "book" - approximately design manual values - then a minimizing is carried out as described above. (The Bdd term in GgammaBr mentioned above is here fixed at zero.) One box of the vertical scale is 0.05 units in Ggamma. We learned above that we need to know the Ggamma to better than half a box throughout the acceleration cycle. We are minimizing the differences between pairs of points, but we need to get these two functions to overlay throughout the acceleration cycle. If the two functions GgammaFr and GgammaBr have different shapes no matter what we do to our coefficients then we can not get to a perfect match. We need to look beyond just the minimization of the least-squares sum and try to figure out how to properly modify the two functions $\operatorname{GgammaFr}(\mathrm{t})$, and $\operatorname{GgammaBr}(\mathrm{t})$ to gain the freedom to get to the same function of time which hopefully is also Ggamma(t). The other two plots below show how the difference reacts if $\mathrm{R} 0$ is changed by $+/-0.8 \mathrm{~mm}$. These changes increase the chisq/deg of freedom from 1.7 to 3 .
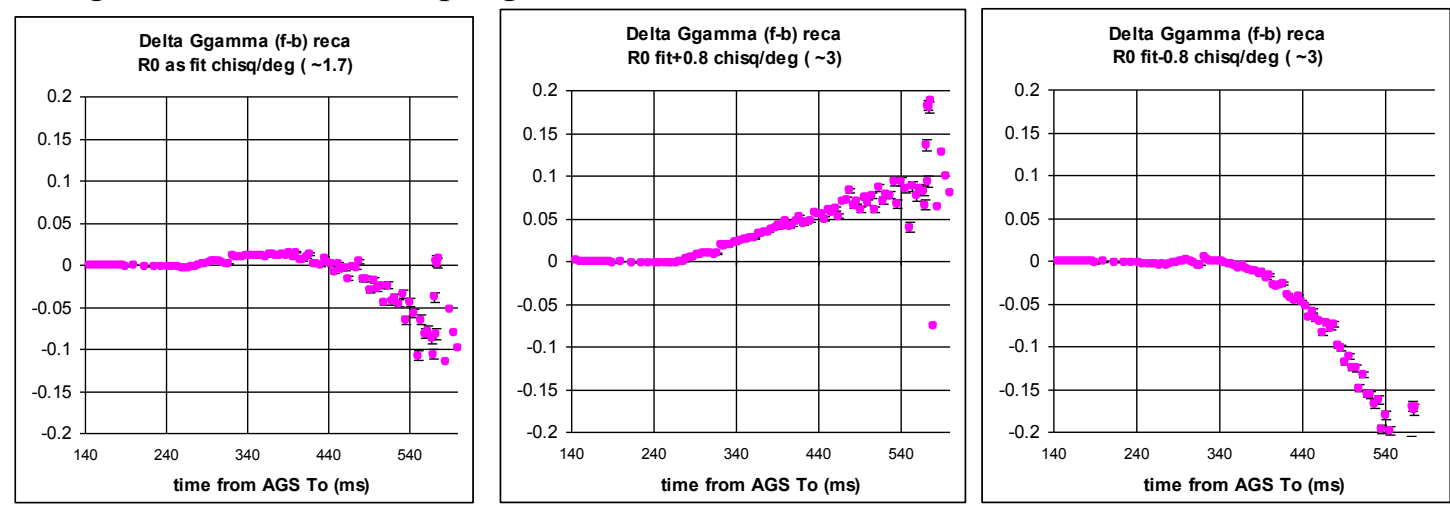

Figure 1: GgammaFr(t)-GgammaBr(t) for a set of AGS data and for (left) a simple fitting, and then center and right: shifting R0 by $+/-0.8 \mathrm{~mm}$ from the original solution. The horizontal scale is time in the cycle, spanning the full acceleration period. The vertical scale is the difference in Ggamma using the two methods of calculation.

Clearly from figure 1, just changing R0 does not get us to a better solution, well it shouldn't. But one plausible conclusion that does follow from the rather violent swinging of the difference curve late is that we probably know R0 late in the cycle to better than 
$1 \mathrm{~mm}$. Then again from the plots, now looking early in the cycle, it is clear that this rather crude radial information allows us to accurately predict Ggamma just from $\mathrm{F}$ and $\mathrm{r}$ and hence to provide the GgammaBr machinery an injection starting value. This will be true provided we have made measurements early in the cycle, the gauss clock starts counting at injection, and $\mathrm{R} 0$ is really a constant throughout the cycle. A $1 \mathrm{~mm}$ change (or uncertainty) in R0 has an insignificant effect on the plotted delta early in the cycle and, since GgammaBr essentially does not depend on R0, therefore has an insignificant effect on GgammaFr. The claim then is that under the right measurement conditions we can read off one of the GgammaBr calibration numbers namely B0 from GgammaFr. There is some more discussion of this topic in the appendix I, looking at standard deviations in the measured quantities and among the Action Items.

Two asides:

Aside 1:

The calibrations being used by the Ggamma meter when the jump quad timing runs are taken (i.e. measuring the horizontal tune and GgammaBr at two times close to each resonance crossing and interpolating to better find the crossing time) determine the quality of those times. If the calibrations that are active when making the jump quad timing set are flawed, the timing set will be flawed. If indeed the calibrations are proper and the jump time set is good but a week later the beam energy time dependence changes (an AGS magnetic field or radius change) or the horizontal tune time dependences changes, then the crossing times will no longer be correct. On the other hand, if a week later the gauss clock calibration parameters or the beam position monitors average position changes, that won't affect the jump timing at all.

Aside 2:

In fact the produced GgammaBr(t) - and not GgammaFr(t) - is used in working out jump quad timings because the gauss clock shows little cycle-to-cycle variation while the frequency required by the GgammaFr(t) has the expected significant statistical fluctuations given the measurement windows. For the Jump Quad timing determination setup the windows between measurements are necessarily small - squeezing in around each crossing time and so the frequency precision for a single measurement is poor. The gauss clock has no problem with close timings. 


\section{Model description and a suggested parameter optimization strategy}

To better understand the challenges to learning the calibration constants, we turn to a simple model for the AGS acceleration cycle. The model assumes a simple main magnet field time dependence (figure 2) - flat injection porch, constant ramping section, and flat extraction porch. We take this $\mathrm{B}(\mathrm{t})$ and translate it into a beam momentum using the "book" value for the AGS main magnets radius of curvature. We are assuming our beam is on the central orbit. This gives us the "true" beam energy or Ggamma vs time. We can also use the $B(t)$ to generate a Gauss clock count $(\mathrm{GCC})$ vs time. To find the GCC we need to set the Gauss Clock calibration - counts per Gauss, which we take as 19.5 , close to the measured value.

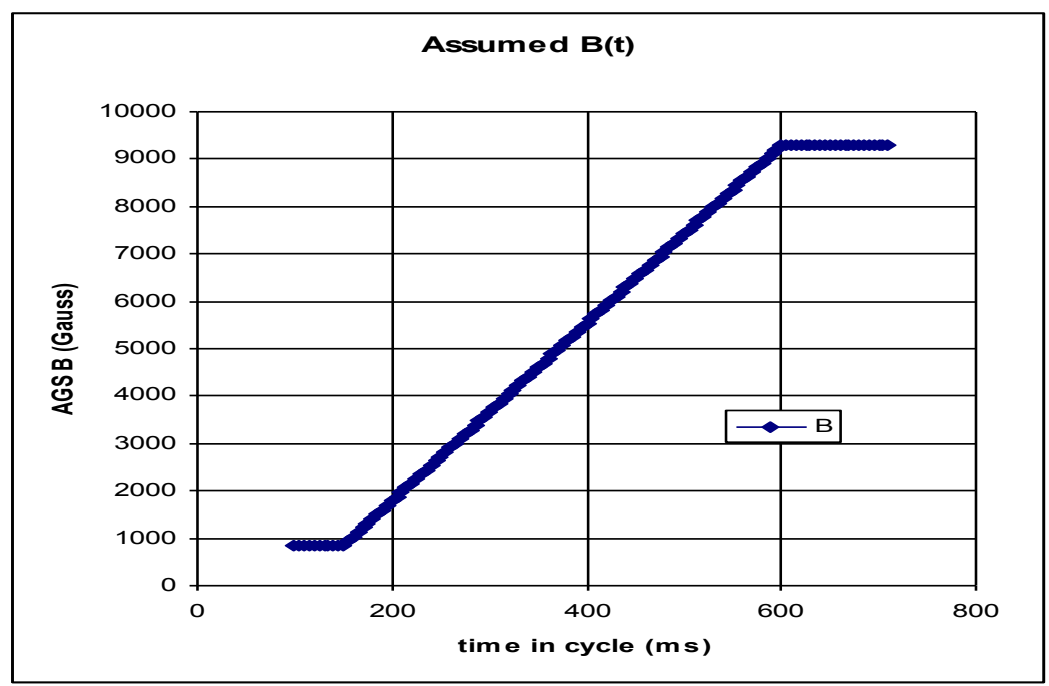

Figure 2: AGS Main magnet time dependence used in this model. The parameters are chosen to mimic in a simple way the actual behavior of the AGS for polarized acceleration.

We can turn the calculated momentum vs time into a revolution frequency vs time. Calculating this frequency requires we know the path length, which is defined as $2 * \pi *$ Ro and we pick Ro to be about what is in the "book" and what is measured, $128453 \mathrm{~mm}$. Again we are on the design central orbit.

Now we can take the tables for GCC(t) and $F(t)$ and with some $r$ (which is the deviation of the beam from the central orbit and starts as zero here) we mimic the Ggamma meter application and calculate GgammaBr and GgammaFr. If we plot these two Ggamma's vs time they are "by construction" identical. If we plot the difference between them or if we plot the difference of either from the "true" Ggamma we get zero.

Next we introduce an error either into the GCC calibration or into the radius such that the reported Ggamma is wrong - the Ggamma error in this example is here chosen to be 0.04. (0.025 was our figure of merit back in section I.) We take this amount of Ggamma error because the errors needed either in the GCcal or in the radius to cause this amount of error in Ggamma are physically realistic. Figure 3 gives the results. To get 
a .04 mistake from GgammaBr we need the GCC calibration to be wrong by .02 . Our model $19.5 \mathrm{GCC} /$ Gauss becomes 19.48 here. The radius needs to be wrong by $0.2 \mathrm{~mm}$. Our model $128453 \mathrm{~mm}$ becomes $128453.2 \mathrm{~mm}$. Though the errors at flat top are equal (by construction) the behaviors during the acceleration cycle are quite different with GgammaBr showing a linear error and GgammaFr curving. This difference in behavior is the only reason we can hope to use this comparison to find the correct values for both factor sets.

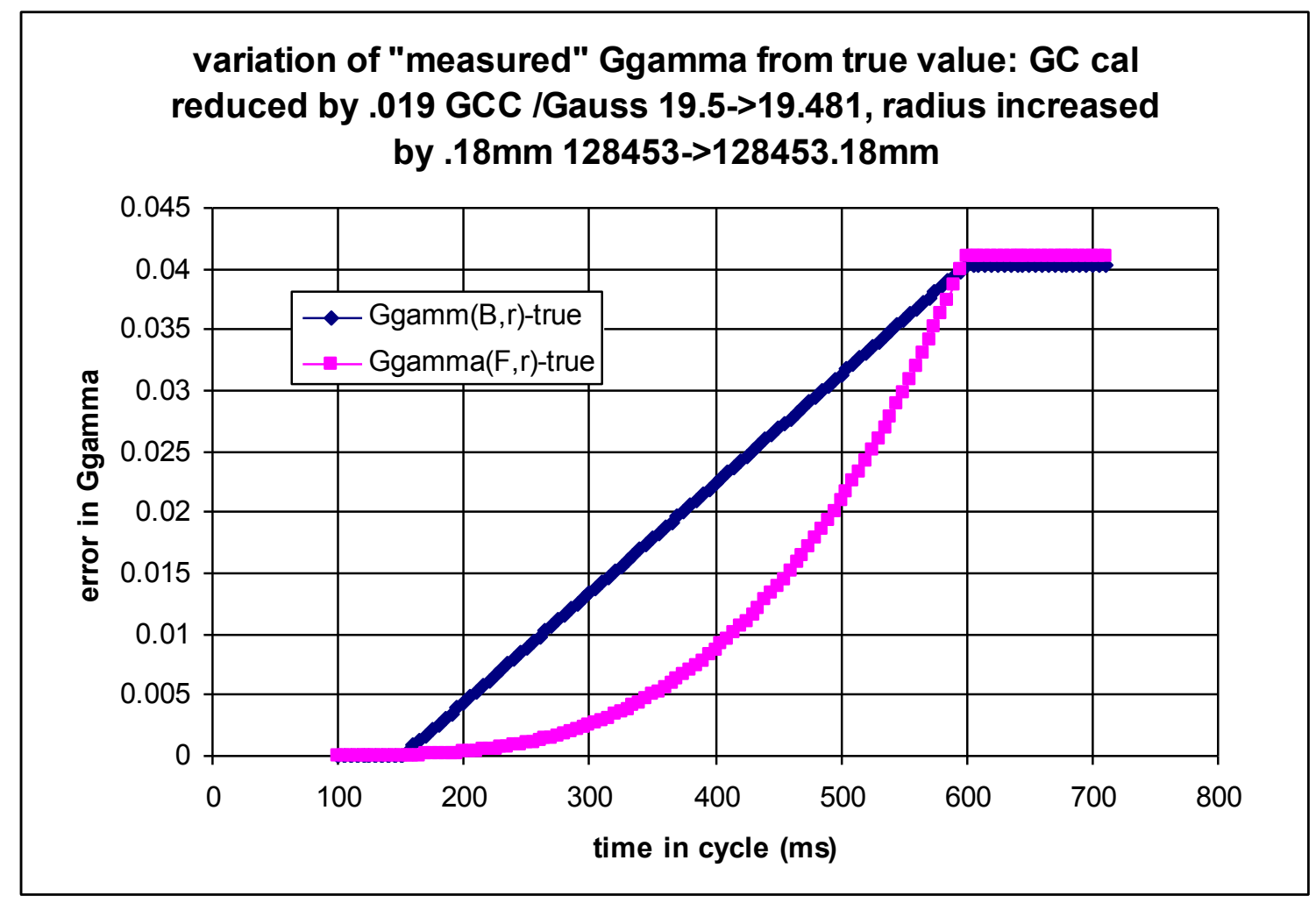

Figure 3: The effects in the calculated Ggammas when errors are introduced into the input parameters.

In an experimental or "beam-based" sense we cannot actually measure these absolute differences. What we can measure is the difference (GgammaFr - GgammaBr) reported by the Ggamma meter. It is then quite possible for example to introduce the magnitude of error just mentioned into the gauss clock through Gcal and a compensating-at-full-energy error into GgammaFr through R0 to restore perfect agreement in this difference late in the cycle. In this case both determinations of Ggamma are wrong throughout the cycle and worst at full energy. The measurable maximum difference between the two energy evaluations during the cycle is unfortunately rather small compared with the true errors, only 0.015 rather than the 0.04 errors at full energy. This is a weakness of the technique if points early and late are much more heavily weighted than points along the ramp. 


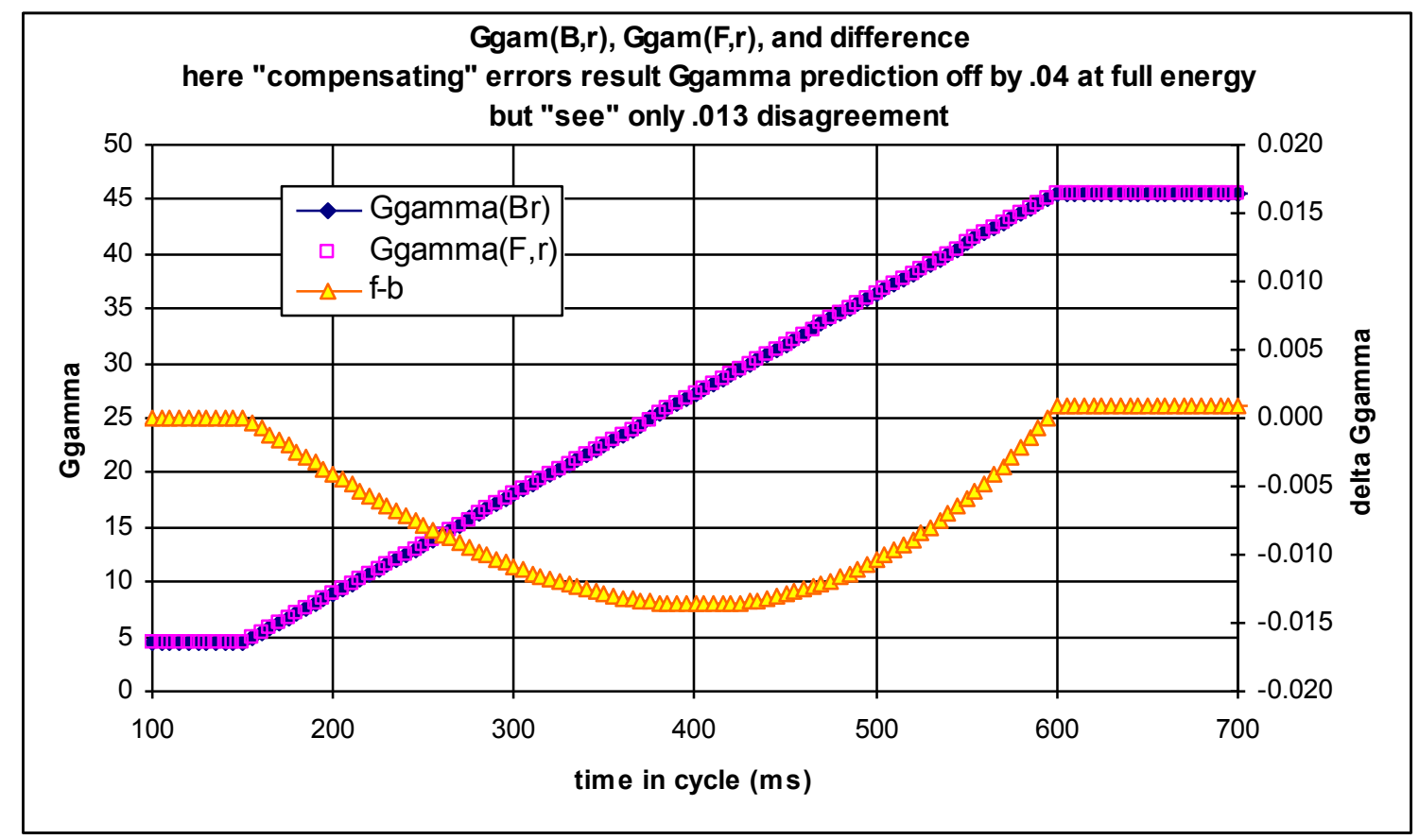

Figure 4: The experimentally available difference between GgammaBr and GgammaFr for the case under discussion

If our "for calibration" set of times samples well the entire cycle and if the systems behaves according to the model then conventional least-squares fitting should work just fine. We saw above that the result for a real set was less than acceptable so this model is surely not complete Nevertheless gaining some intuition from the model is useful. In particular we see that an error in GCcal will result in a linearly growing difference error with energy while an error in $\mathrm{R} 0$ will result in a difference that grows quite nonlinearly with energy. Simply plotting against energy rather than time should give a more useful graphic for manual fitting. And getting the difference down to very small numbers but only over a subset of the full acceleration range carries no guarantee of the quality of the result. In particular the measured residual difference can significantly underestimate the true error.

From the preceding a strategy for "fitting" the Ggamma calibration factors to compete against the simpler-to-implement least squares fitting emerges. The curvature in the difference (figure 4) results from using an erroneous R0. Provided such curvature is visible in the read data, small adjustments in R0 should bring the difference to be a straight line. The slope of that line will then reflect any error in the GCcal and again small adjustments to GCcal can be applied to bring the slope to zero. 


\section{Real data study}

\section{A. The machinery - the logged data and spreadsheet analysis being used. One data set analysis in detail.}

The machinery in use - by an example:

The procedure here is to gather together data sets logged (LogView) by the Pet page "Ggamma Meter" which include the calculation inputs (frequency, raw gauss clock counts, corrected gauss clock counts, radius, calibration numbers) and also include the calculation results from the Ggamma meter (GgammaBr and GgammaFr for each measurement time in each AGS cycle in the sets. We can then recalculate GgammaBr and GgammaFr using different calibration numbers for the gauss clock and for the value of R0.

We start by looking at a single cycle (from 23May13 10:02:26).

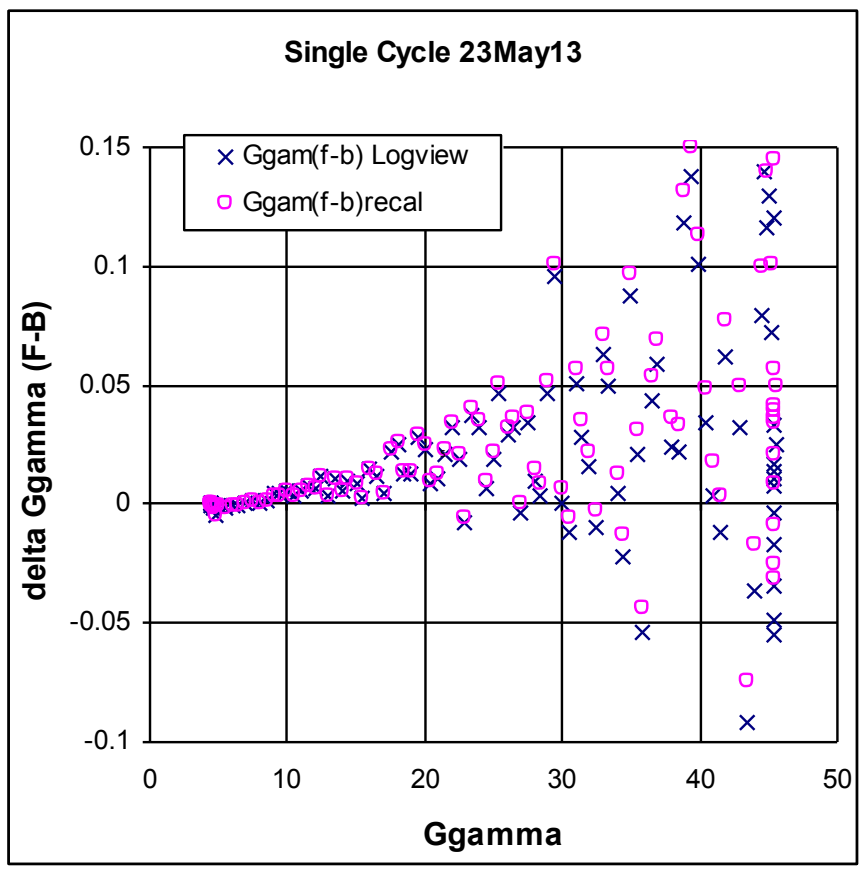

Figure 5a: GgammaFr -GgammaBr Logview vs recal (as found)

Here the horizontal scale is the beam energy (Ggamma) where either Ggamma evaluation is precise enough to be used. The vertical scale is the difference in the calculated values of Ggamma. The black x's come directly from the LogView data and the magenta circles are recalculated Ggamma values using the inputs also found in the LogView data sets.

The scatter of the data is huge. At first we fail to get good agreement (figure 5a). The recal and Logview data show systematic differences. We find the values for $\pi$ and $c$ in the Ggamma meter analysis are very slightly different from values used in our recalculation (which values were found with Google's help and so must be right...). 
Historically these differences are not really problems - sanity aside - because only the product $(\pi)(\mathrm{Ro}) / \mathrm{c}$ matters in calculating GgammaFr and Ro is free (in the fitting) to accommodate the tiny differences we are mentioning. Putting the same (less right) values for $\pi$ and $\mathrm{c}$ into the recal machinery gets us to figure $5 \mathrm{~b}$ - satisfactorily close agreement.

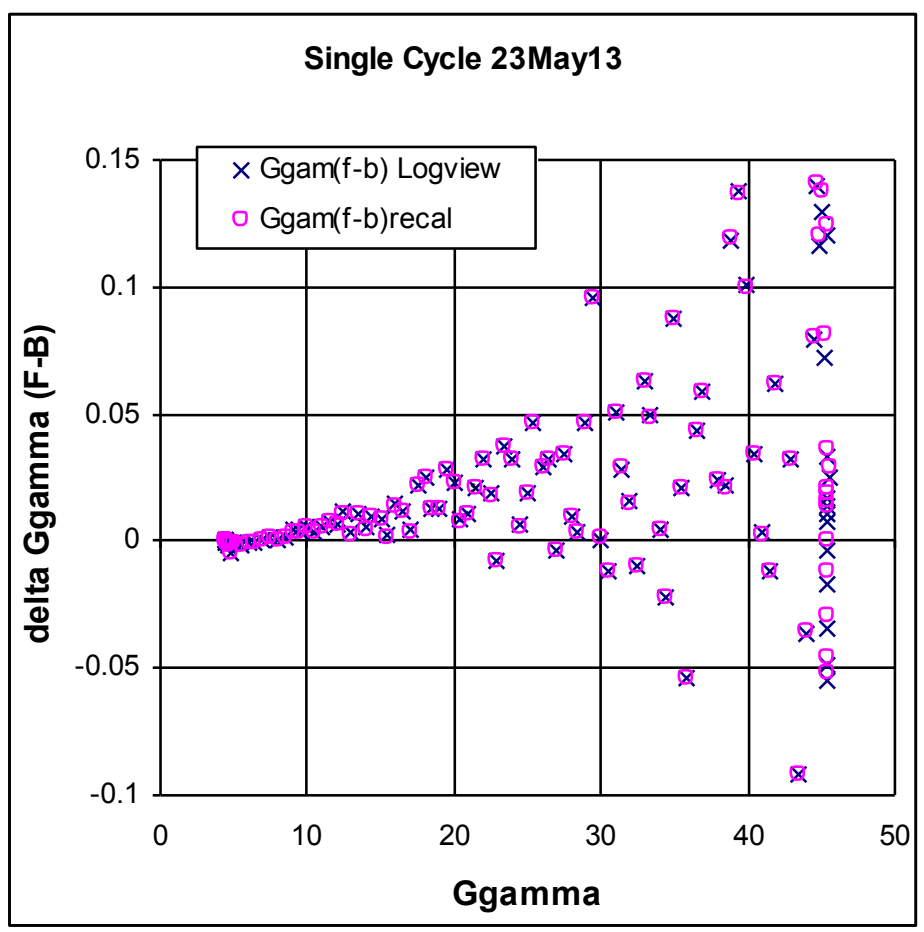

Figure 5b: 1 cycle - Same as 5a) except put Ggamma meter values of $\pi$ and c into model

Up to now we have been using the "corrected GCC" from Labview. The difference between raw GCC and corrected GCC has to do with the Bdd parameter that we have not discussed. This correction will be treated later but we mention it now to try and reduce confusion later. We want to be able to revisit this correction in the recal to allow changing the Bdd parameter. We follow the Pet page machinery that does the Bdd correction - in spirit at least - and indeed replicate figure $5 \mathrm{~b}$ now with our recalculated gauss clock counts, taking into account the second derivative of $\mathrm{B}$.

Next we try to reduce the scatter by averaging over many (349 here) cycles. (Data set 23may13, 10:02:22 - 10:24:56). One obstacle to getting good result is the occasional cycle with "bad" data, and we do not want to include even one of these in the averages. Usually the bad cycles are cycles when beam does not survive. Certainly these can be removed. With more data figure 5 morphs to figure 6 . The scatter (standard deviation of the LogView data divided by the square root of the number of cycles taken) is nicely reduced but is still significant at high energy. The scatter late in the acceleration cycle does not go away with more statistics. 


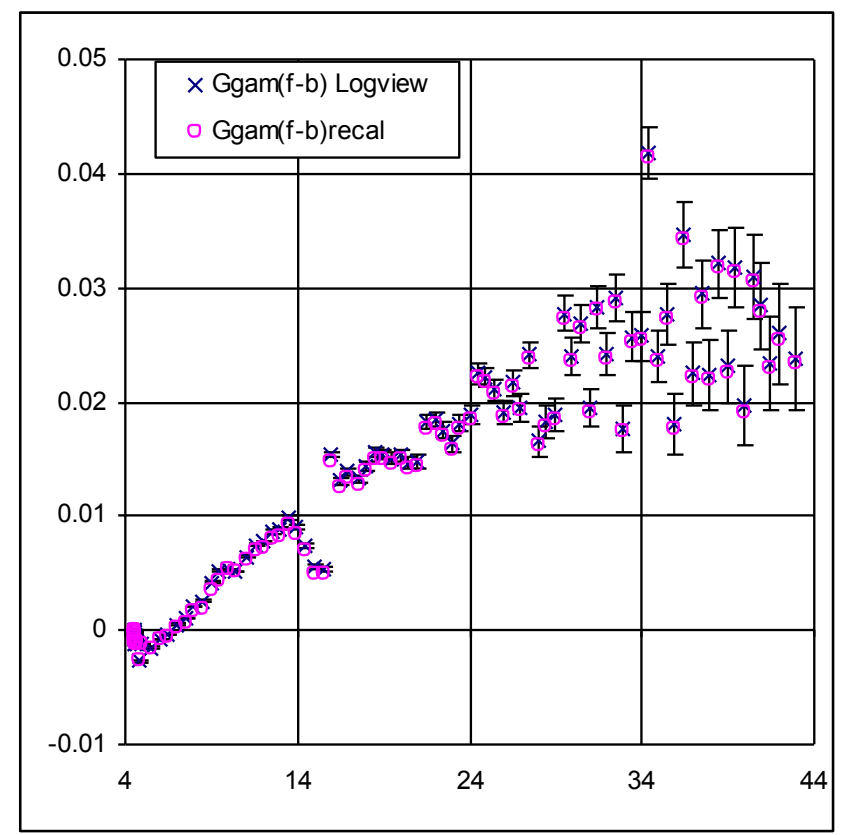

Figure 6a: 349 cycles GgammaFr-GgammaBr

Now we want to work with this data set, changing the Ggamma meter parameters that generate it to see what the Ggamma results are with different coefficients. The magenta points in figure 6a show the result of reanalysis with unchanged parameters. We take this as success as far as being able to now revisit the parameters. The initial parameter set is $(\mathrm{GCcal}, \mathrm{R} 0, \mathrm{~B} 0, \mathrm{Bdd})=(19.494,128453.2,846,-10)$ with the units left off. With $\pi$ and c set to more correct values, we continue working with this data set.

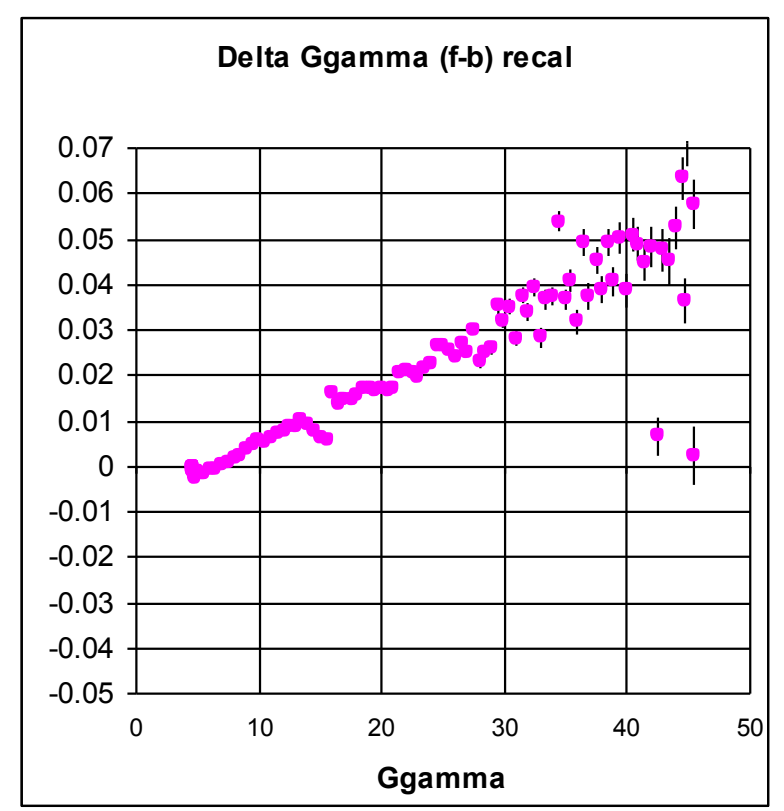

Figure 6b: Differences now with correct $\pi$ and $c$, just the recalculated data points. Fit chisq/d 2.9. 
Figure 6c shows the same data using the "book" parameters (GCcal, R0,B0, Bdd $)=(19.5,128453,846,0)$

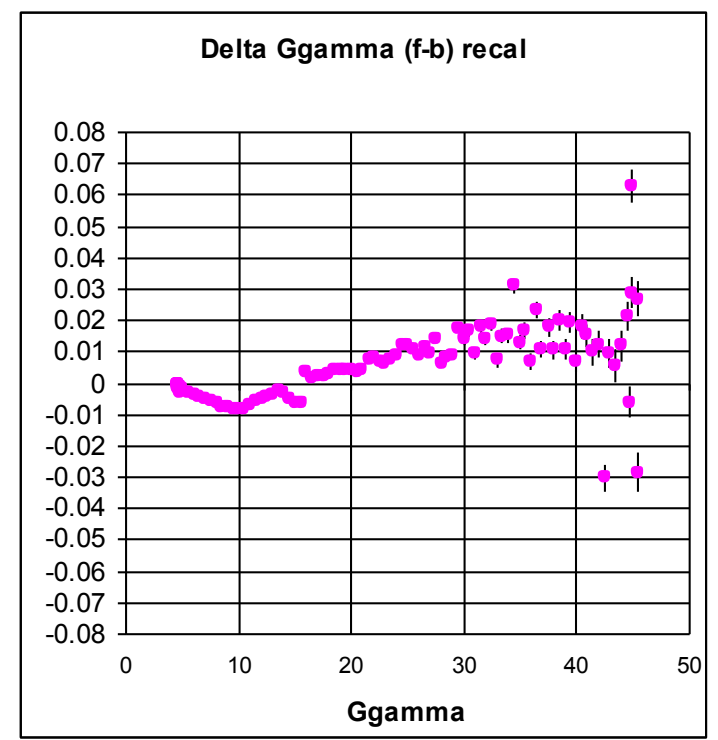

Figure 6c: Book parameters (chisq/d 3.5)

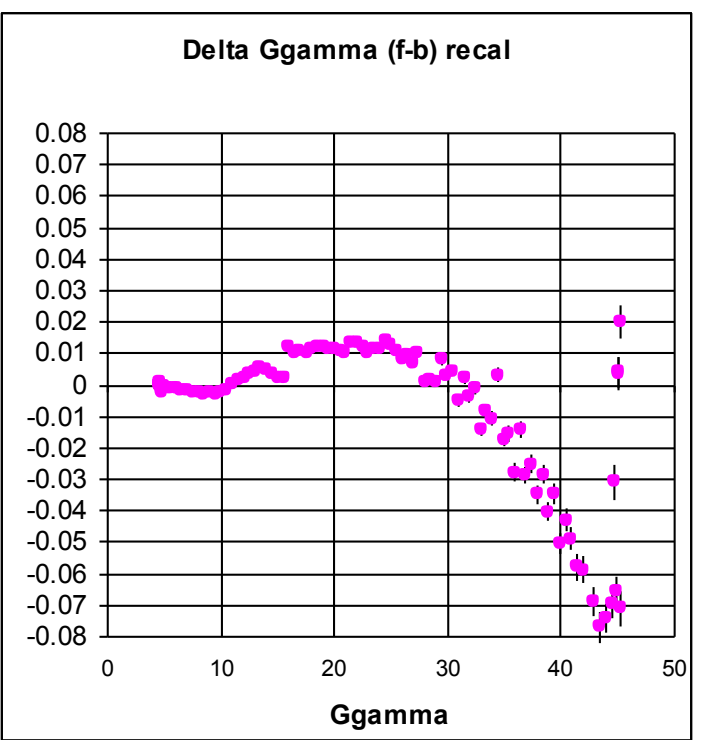

Figure $6 \mathrm{~d}$ fitting, noBdd, chisq/d $\sim 1.76$

Applying an automatic fitting using "book" starting parameters including no Bdd term takes us to figure $6 \mathrm{~d}$ above. The tiny error bars early and large error bars late are forcing the fitting contrary to "eye" expectations.

At this point we will finally specifically define the Bdd term. The cusp in the data in both of the above plots near Ggamma 10 is not a feature of (cannot be coped with by) the simple model. Because this feature occurs about when the slope of the main magnet field voltage is changing - between $\sim$ zero and the approximately constant value achieved during most of the acceleration ramp - an ad hoc correction term proportional to this slope change is applied to the instantaneous GCcal, namely GCcal $(\mathrm{t})=$ GCcal $\left.{ }^{*}\left(1+\mathrm{Bdd}^{*} \mathrm{~d}^{2} \mathrm{~B} / \mathrm{dt}^{2}\right)\right)$. Adding this freedom to the fitting produces figure $6 \mathrm{e}$.
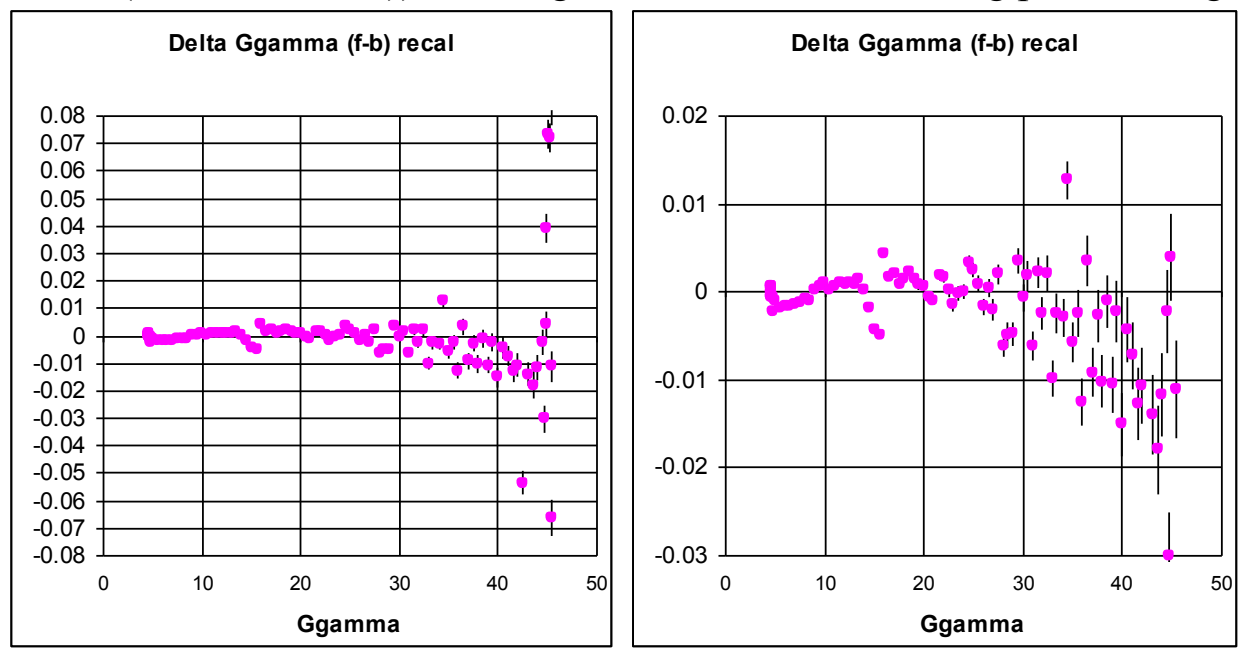

Figure 6e: Allowing GCcal( $\mathrm{t})=\mathrm{GCcal} *\left(1+\mathrm{Bdd}^{*} \mathrm{~d}^{2} \mathrm{~B} / \mathrm{dt}^{2}\right)$, replot the same data with two vert scales 
The Chisq/d drops to 1.1, and the cusp is reduced - by construction. The fitted parameters are $(\mathrm{GCcal}, \mathrm{R} 0, \mathrm{~B} 0, \mathrm{Bdd})=(19.474,128453.07,845.8$, and -10.4) The huge scatter late in the cycle is not improved by this maneuver, but now in the expanded view (ignoring the huge scatter stuff) we can examine the fine smooth structure in the data. We can look for the curvature in this difference as expected from the section II model. Figure 7 shows this same data with fitted parameters except with R0 moved around by $0.2 \mathrm{~mm}$. The curvature from the model appears, perhaps.

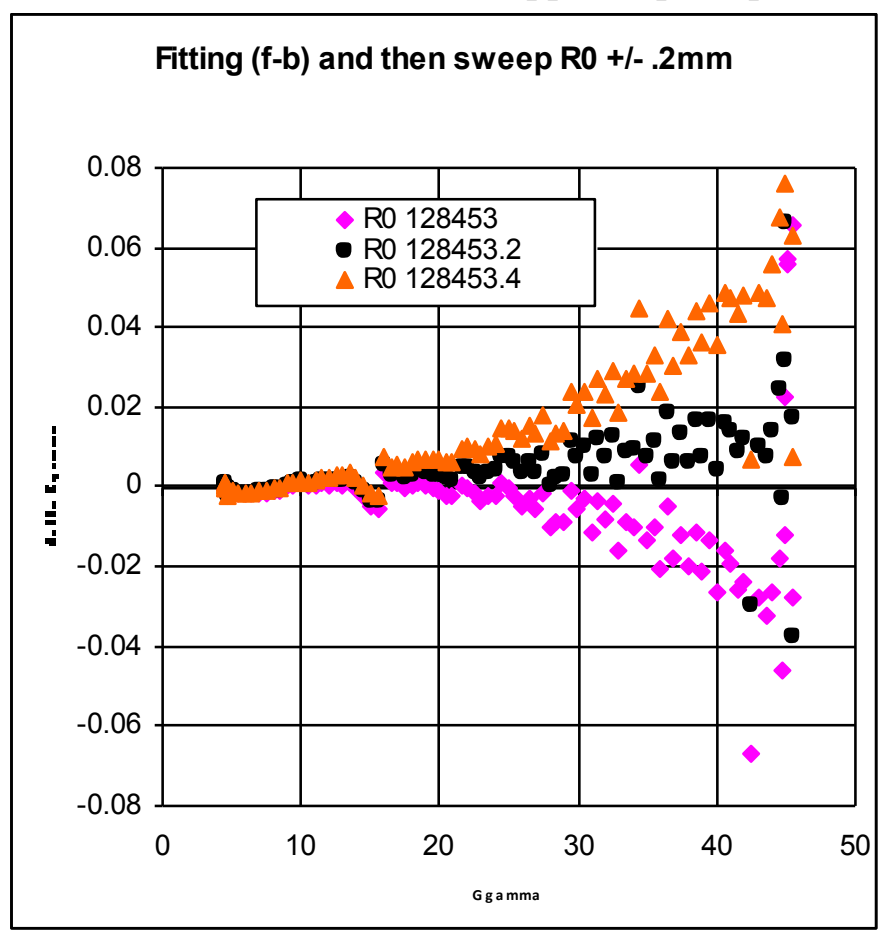

Figure 7: Difference data with R0 shifted in $0.2 \mathrm{~mm}$ steps, all else held fixed.

Following the model, having adjusted R0 to give a straight scatter (here 128453.2) the slope can then brought to zero with GCcal (from 19.475 down to 19.469. 


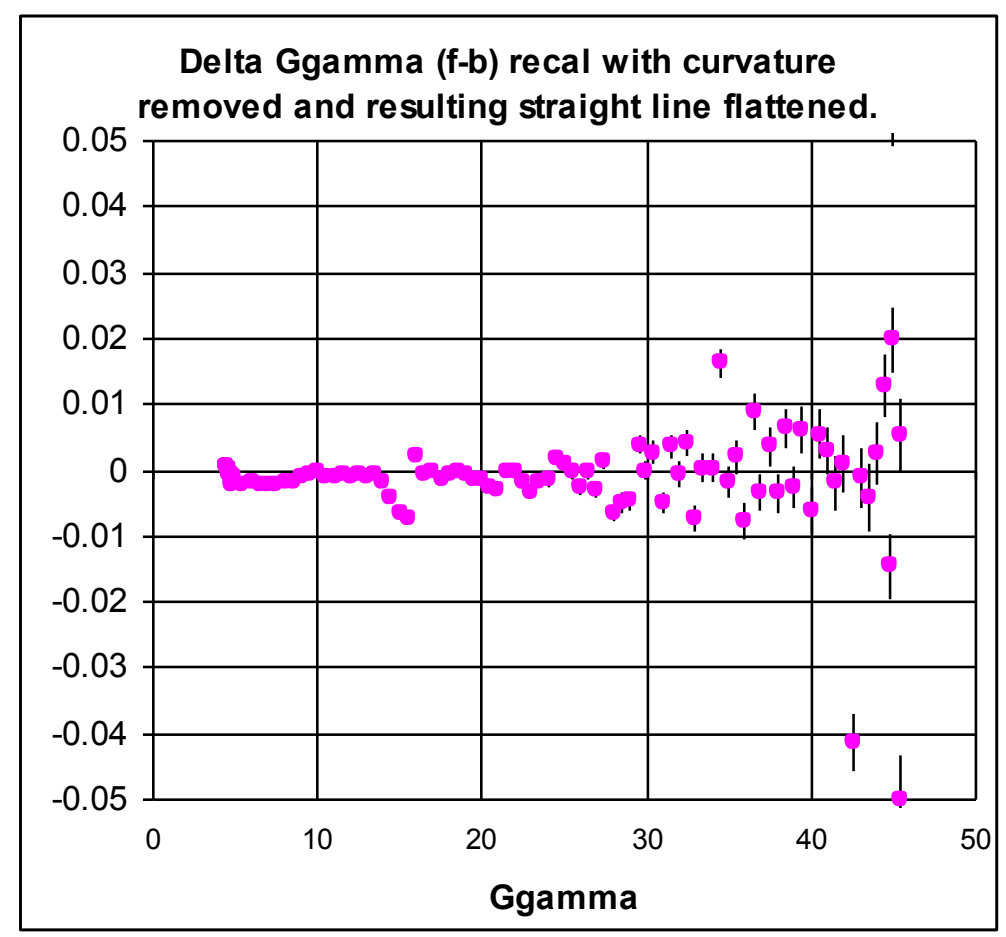

Figure 8: Removing curvature and flattening by hand.

The resulting solution has GCcal $=19.469$ compared to 19.475 . The chisq/d has increased from 1.1 to 1.22. The difference in the predicted GgammaBr for the two sets of calibration factors is given in figure 9 and remains below 0.012 , which is about half of our figure of merit.

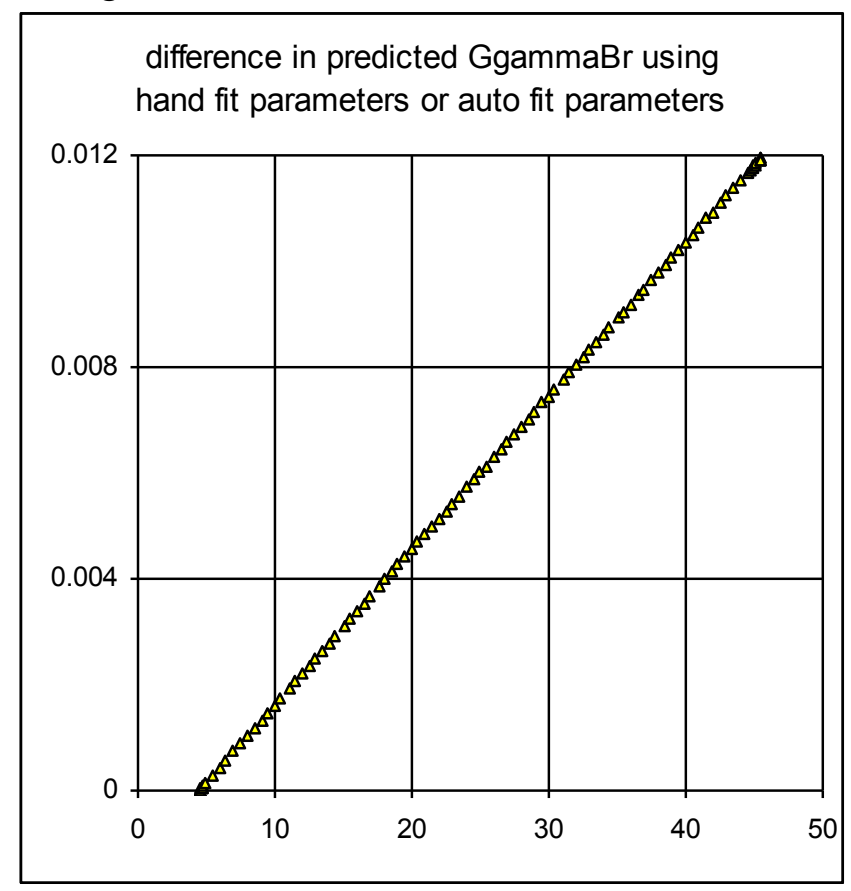

Figure 9: The difference in the predicted Ggamma using auto fit parameters or decurve and flatten parameters, and using the Bdd correction given by the fit. 
The difference in Ggamma of 0.012 at Ggamma 45 would correspond to a disagreement in crossing time of about $(0.012) *(500 \mathrm{~ms} / 50)=120 \mathrm{usec}$. Perhaps this can be tested with the $36+$ intrinsic region. We would need time predictions at the $100 \mathrm{usec}$ level. And of course the GCcal active in the machine for the ineffective jump timing set created in mid-May was 19.494, according to this too high by at least 0.02 and hence expected to provide poor jump quad timings.

This manual fitting ignores the points near the end of the acceleration cycle that are way off the line, plus and minus. If we are to believe the answer, we need to figure out why these points can be discredited. Some associated information is given in the next figures. The Ggamma differences and the Ggamma values themselves are plotted in figure 10. This dance occurs just around the higher energy resonances most needing good jumping times. The reported standard deviations of the input data are plotted in figure 11 . There is little indication of any problem with the data until $585 \mathrm{~ms}$, which is after our problem. We do not yet have a reason to discard these points.

One possible explanation for the difference between GgammaFr and GgammaBr here is that the orbit length is changing in ways invisible to the measured radial average e.g. in the vertical plane. No plausible numbers for such an effect have been figured out; the required orbit distortions appear to be unphysical..

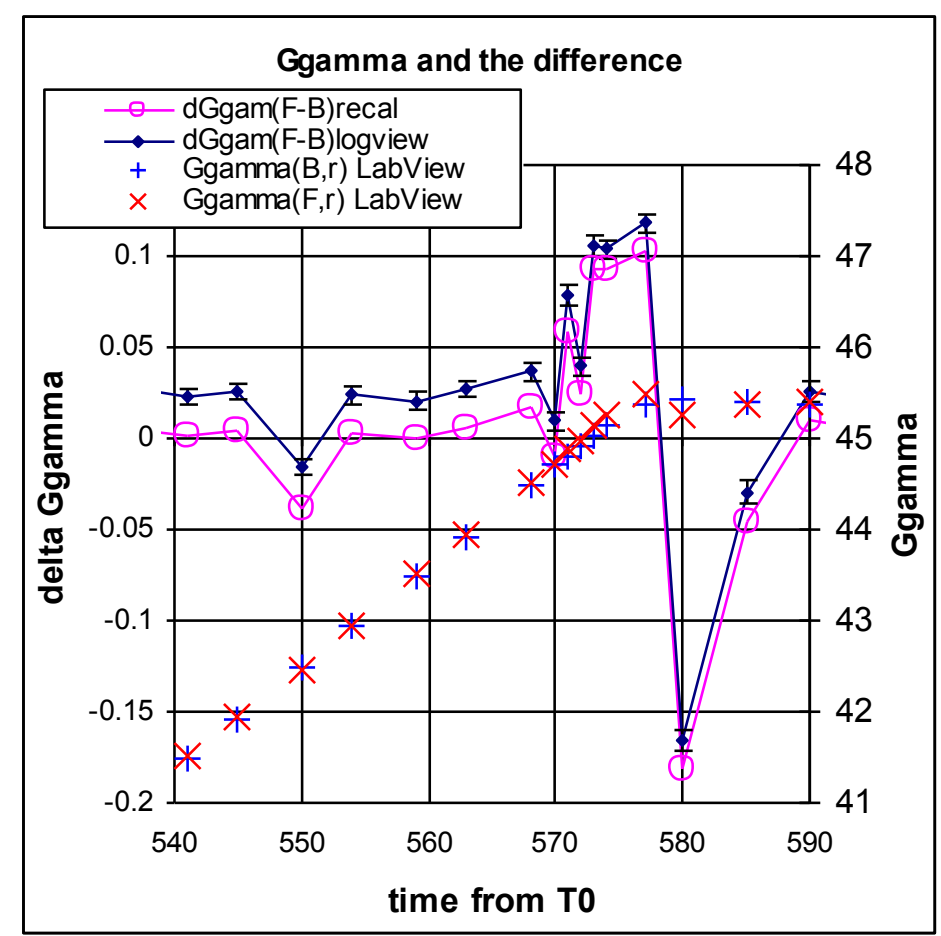

Figure 10: Logview and recalculated Ggamma differences, and the Logview data. 


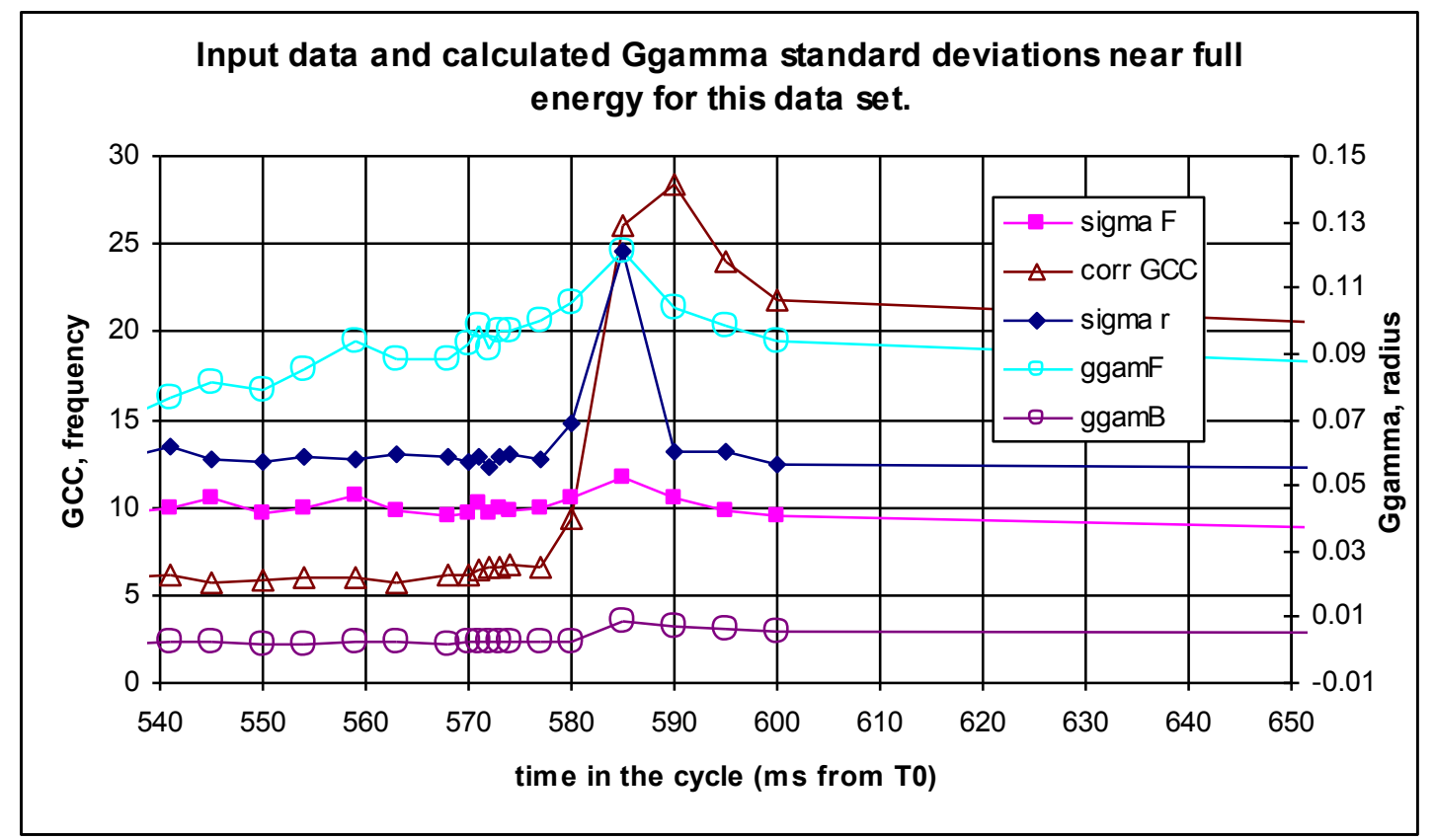

Figure 11: The standard deviations in the inputs to the calculations, and in the Labview answers 


\section{B. 2013: Some example sets and parameters summary plots}

Returning to the data sets, it is at least instructive to apply these machineries to several data sets over the 2013 running period. In each case the procedures remain the same as above. Both an automatic fitting and a "decurve" and flatten fitting are tried. It is found to be necessary to sometimes ignore points taken out on the flattop and so if such points exist, they are always excluded from the data used. Again this is not understood, especially since the points sometimes fall right on prediction and other times are not close.

The auto-fitting uses "book" starting values. Figure 12 gives the resulting GCcal throughout the run. Also shown is the value in the Ggamma meter - the value used for any new Jump Quad timing attempts - and, just for reference, our 200 usec figure of merit time precision need. The eye fitting -reducing curvature and then flattening - tends to result in a value for GCcal slightly lower that that returned by the auto-fitting. And the time structure is about the same. It would appear that the gauss clock changed sometime in early May and perhaps with some rather fast time structure in late May. In as much as this behavior is not understood and significantly affects the time generation, it would seem necessary to require the calibration be updated whenever a new timing is to be made.

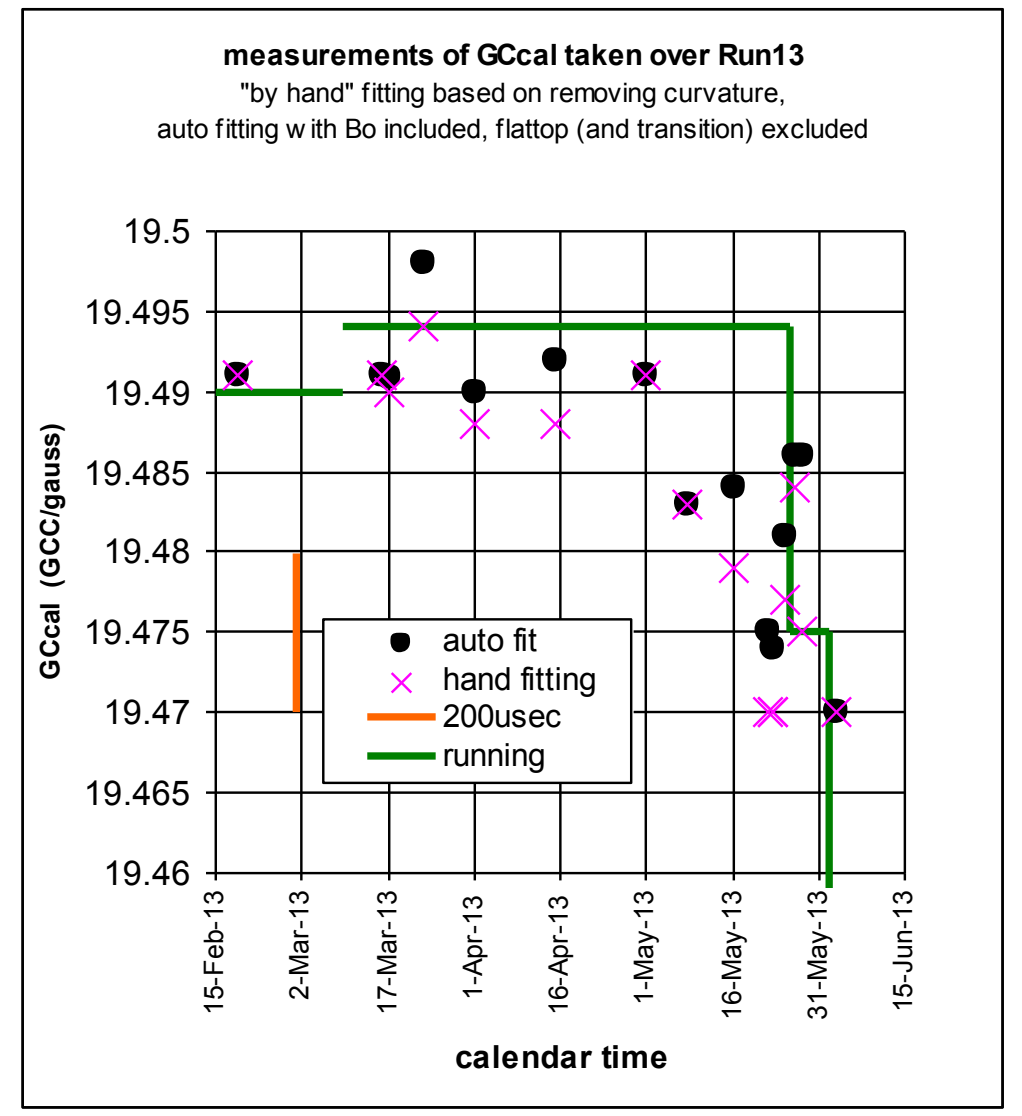

Figure 12: Calculated GCcal for clumps of data throughout Run 13.

Figure 13 gives the same information but now for the calculated circumference. 


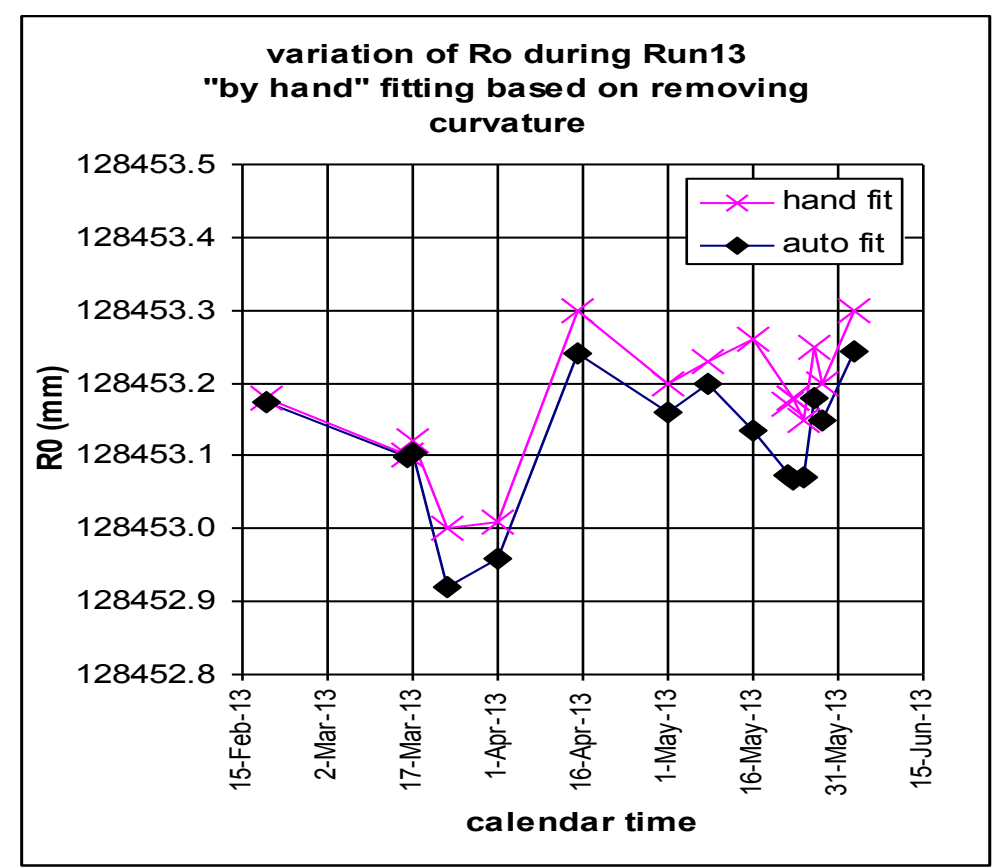

Figure 13: Ro as derived from manual and auto fitting Run 13

The manual fitting tends to give a value for Ro larger than that from the auto fitting by $0.1 \mathrm{~mm}$. Of course this is not directly involved in the timing generation given that we use GgammaBr, but the result encourages that there is some consistency to this drill.

The next plot gives the starting field for the gauss clock as calculated by the two methods. Again there is a consistent small disagreement and again this is basically encouraging. In this case there is a better way to get this information but only if we slightly modify the Ggamma Meter machinery in the future. The information from frequency and radius is accurate enough at proton injection to tell the energy there. Provided that for the Ggamma meter we start the gauss clock counting at injection and make a beam frequency measurement there, we can calculate $\mathrm{B} 0$. In the present machinery, the gauss clock starts counting at AGS T0. There are then about $150 \mathrm{~ms}$ between start and injection when the gauss clock is accumulating up and down counts. The field is nominally flat with some ripple during this period and the calibrations of up and down are nominally equal, but we do not need to assume either of these ideas if we just start counting at injection. Then B0 can be pulled from the fitting which would be good. Indeed then also we can track the injection field - which would be useful in its own right. 


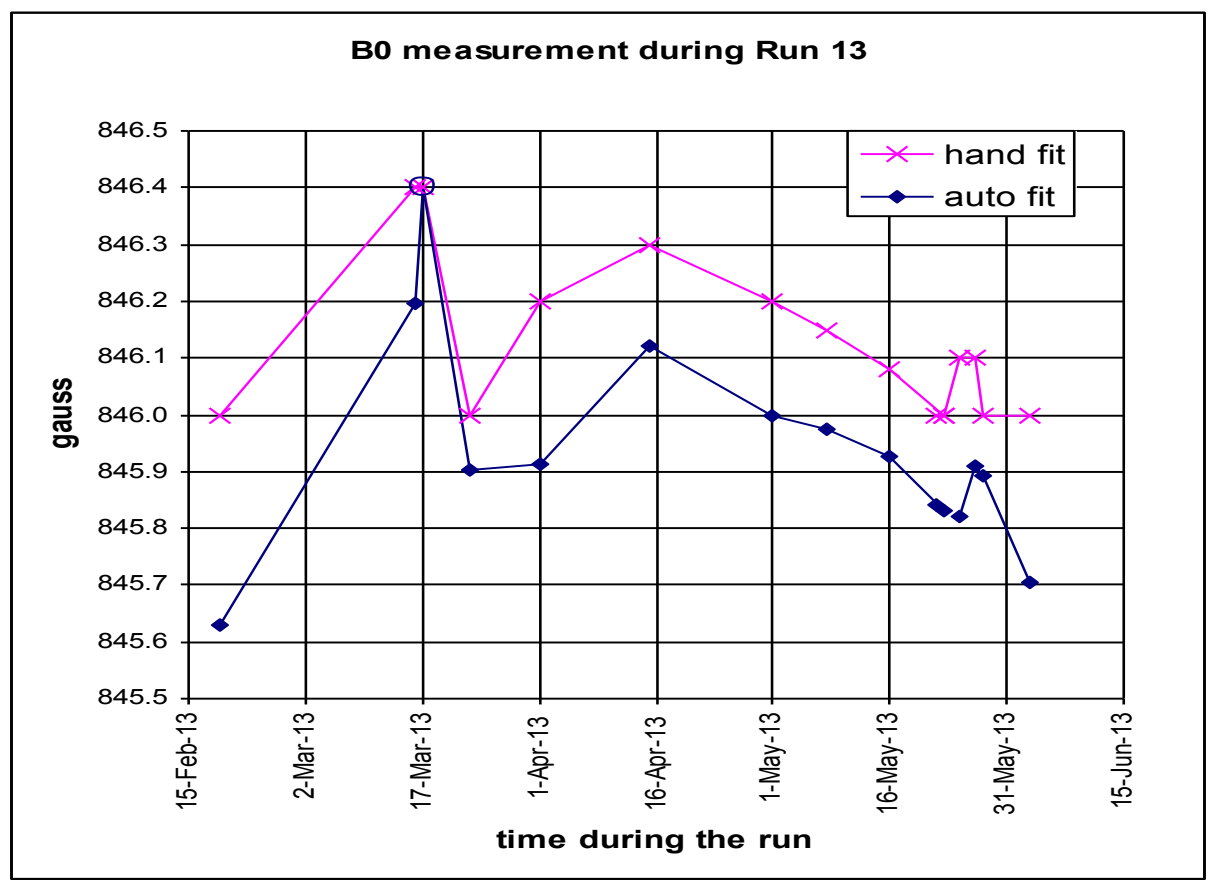

Figure 14: B0 as calculated from the hand and auto fittings during Run 13

And finally the time dependence of the Bdd parameter is given in figure 15 . This is the least well motivated of the fitting parameters and indeed for the hand fitting there is not a clear prescription on how to set it. For some data sets its value is highly constrained - removing the cusp - while for others the cusp morphs into mushy structure. The value coming from the auto-fitting has more meaning. It is satisfactory that this fitting works well starting with the value zero. The hand fitting also starts from zero, but is sometimes not well constrained.

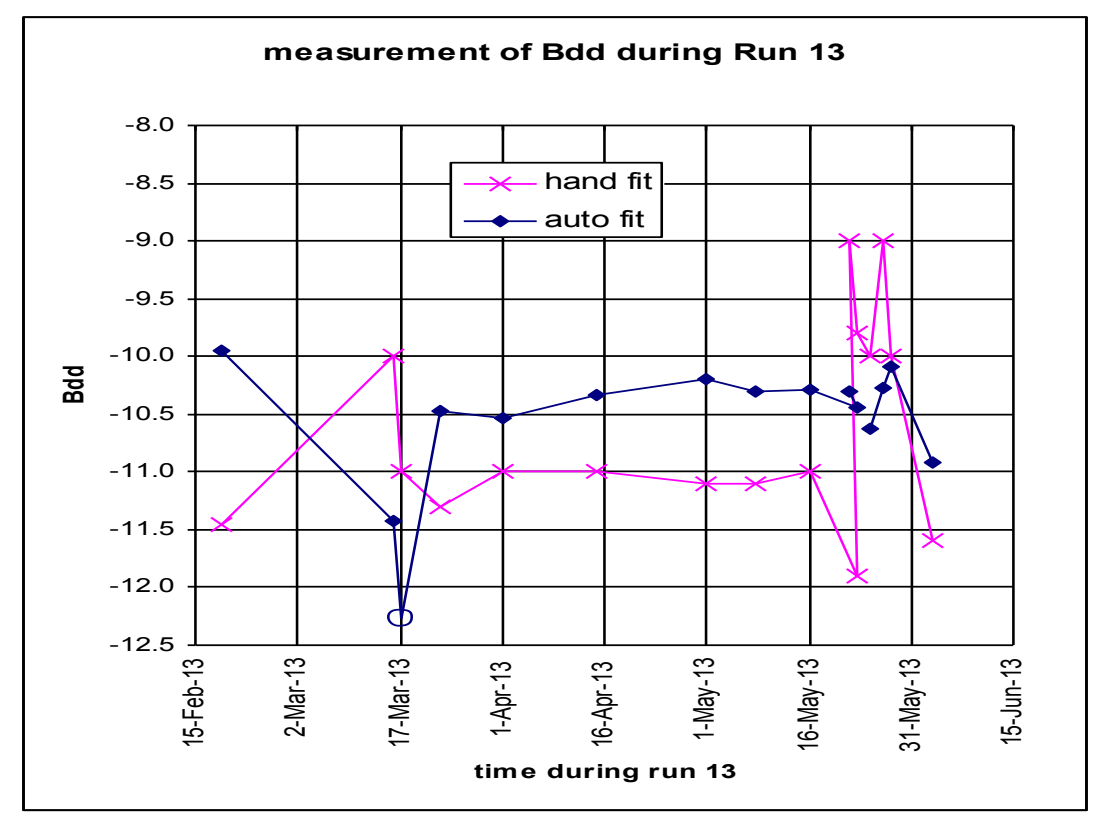

Figure 15 The Bdd fitting parameter during Run 13 as calculated by the two fittings 
(choose no curvature over minimum chisq) Hand fitted results

\begin{tabular}{|c|c|c|c|c|c|c|c|c|c|}
\hline cycles & & $\begin{array}{l}\text { Gccal } \\
\text { counts/ } \\
\text { gauss }\end{array}$ & $\begin{array}{l}\text { R0 } \\
\mathrm{mm}\end{array}$ & $\begin{array}{l}\text { B0 } \\
\text { gauss }\end{array}$ & Bdd & $\begin{array}{l}\text { Gccal } \\
\text { counts/ } \\
\text { gauss }\end{array}$ & $\begin{array}{l}\text { R0 } \\
\text { Mm }\end{array}$ & $\begin{array}{l}\text { B0 } \\
\text { gauss }\end{array}$ & Bdd \\
\hline & 5-Jan-12 & 19.489 & 128453.20 & 844.50 & -6.50 & 19.488 & 128453.216 & 844.420 & -6.370 \\
\hline & 25-Mar-12 & 19.477 & 128453.43 & 845.00 & -8.50 & 19.475 & 128453.460 & 845.050 & -9.050 \\
\hline & 25-Mar-12 & 19.479 & 128453.40 & 845.00 & -8.00 & 19.475 & 128453.470 & 845.100 & -9.040 \\
\hline 181 & 19-Feb-13 & 19.491 & 128453.18 & 846.00 & -11.45 & 19.491 & 128453.174 & 845.630 & -9.953 \\
\hline 230 & 16-Mar-13 & 19.491 & 128453.10 & 846.40 & -10.00 & 19.491 & 128453.098 & 846.197 & -11.430 \\
\hline & 17-Mar-13 & 19.490 & 128453.12 & 846.40 & -11.00 & 19.491 & 128453. & 846.400 & -12.270 \\
\hline 152 & 23-Mar-13 & 19.494 & 128453.00 & 846.00 & -11.30 & 19.498 & 128452.920 & 845.903 & -10.480 \\
\hline 230 & 1-Apr-13 & 19.488 & 128453.01 & 846.20 & -11.00 & 19.490 & 128452.960 & 845.913 & -10.534 \\
\hline 228 & 15-Apr-13 & 19.488 & 128453.30 & 846.30 & -11.00 & 19.492 & 128453.240 & 846.120 & -10.335 \\
\hline 230 & 1-May-13 & 19.491 & 128453.20 & 846.20 & -11.10 & 19.491 & 128453.160 & 846.000 & -10.200 \\
\hline 167 & 8-May-13 & 19.483 & 128453.23 & 846.15 & -11.10 & 19.483 & 128453.198 & 845.975 & -10.299 \\
\hline 230 & 16-May-13 & 19.479 & 128453.26 & 846.08 & -11.00 & 19.484 & 128453.135 & 845.927 & -10.294 \\
\hline 231 & 23-May-13 & 19.470 & 128453.18 & 846.00 & -11.90 & 19.474 & 128453.069 & 845.830 & -10.445 \\
\hline 349 & 23-May-13 & 19.468 & 128453.20 & 846.10 & -11.00 & 19.475 & 128453.074 & 845.840 & -10.298 \\
\hline 244 & 25-May-13 & 19.477 & 128453.15 & 846.10 & -10.00 & 19.481 & 128453.070 & 845.820 & -10.630 \\
\hline 227 & 27-May-13 & 19.484 & 128453.25 & 846.10 & -9.00 & 19.486 & 128453.180 & 845.908 & -10.275 \\
\hline 228 & 28-May-13 & 19.475 & 128453.20 & 846.00 & -10.00 & 19.486 & 128453.150 & 845.894 & -10.088 \\
\hline 150 & 3-Jun-13 & 19.470 & 128453.30 & 846.00 & -11.60 & 19.470 & 128453.243 & 845.706 & -10.918 \\
\hline
\end{tabular}

fitting, no flattop points, no transition jp pts B0 takes part in the fitting, start at "book" book:

$\begin{array}{llll}19.5 & 128453 & 846 & 0\end{array}$

A Table of the calculated calibration constants throughout Run 13 


\section{2012: An experimentally poor JQ timing set generation, associated $\mathrm{JQ}$ on and off data, fitting issues}

Now we turn to some data taken during the 2012 proton run - so a year earlier than where we have been. A Jump Quad timing set was generated on March 25, 2012. The data taking was mostly carried out by Rocco Tuccio. AGS final polarization was measured (by Haixin Huang and the author) over many "switchings" between the new timing set and the active timing set. The new set was clearly inferior. This gives another opportunity to see if we can understand from the calibration constants in play at that time (for which the author was responsible) the poorer performance of the new timing. To some extent there is no surprise here. We will see that the calibrations were not optimal by the techniques used now although if this were the whole story the deterioration caused to the measured polarization seems somewhat large. Another reason for looking at these sets is that calibration data is available both with the JumpQuads pulsing and with the JumpQuads turned off. These sets allow asking directly if the late-in-the-cycle jumping around of the differences between GgammaBr and GgammaFr is due to the JumpQuads. The answer is no.

We have two set of data collected during the timing data acquisition 25Mar12, figure 12a with the JQs pulsing and 12b with the quads off. These Ggammas are generated using the calibration numbers in play for the timing calculation. The calibration setup in use were: $(\mathrm{GCcal}, \mathrm{R} 0, \mathrm{~B} 0, \mathrm{Bdd})=(19.49,128453.5,846.2,-11)$.

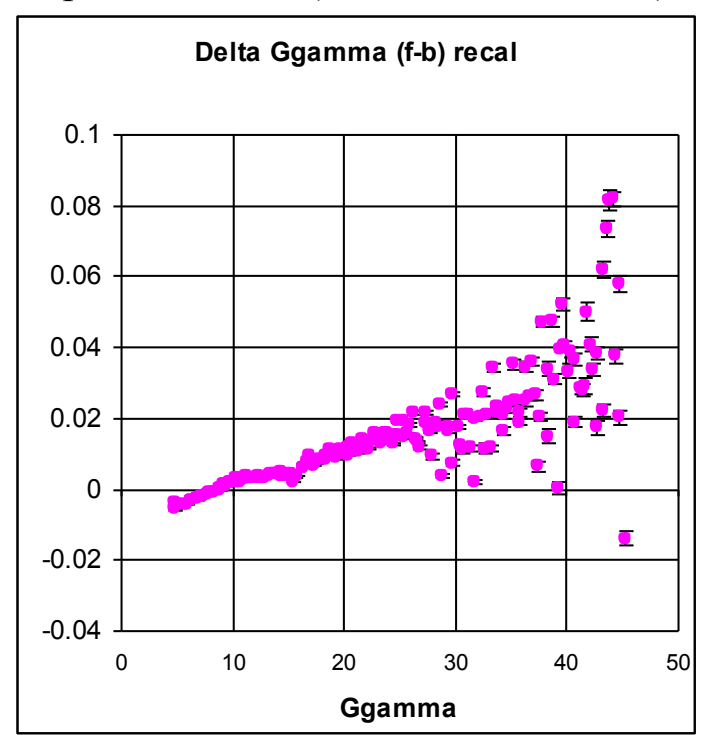

Figure 12a: difference JQ on chis/d 2.65

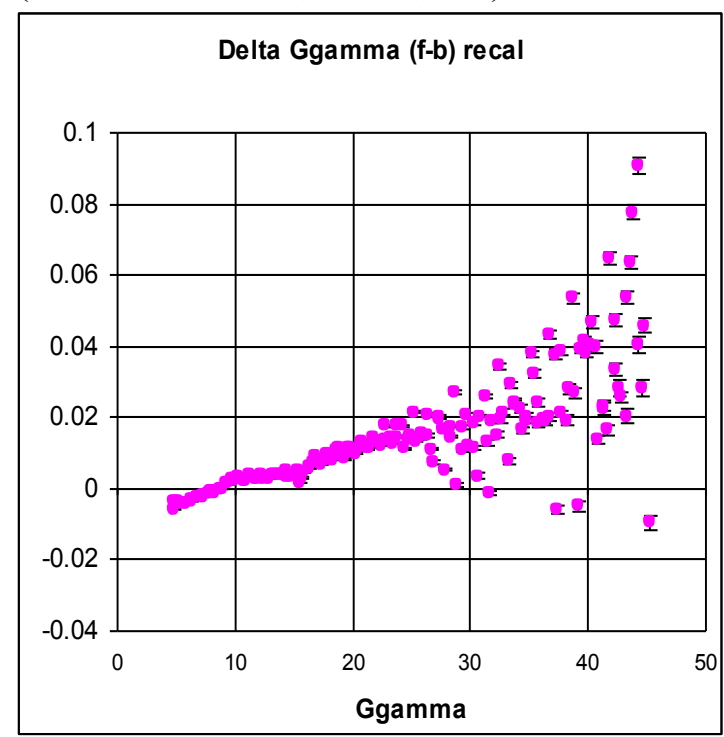

Figure 12b: difference JQ off chisq/d 2.76

First you might ask why weren't the calibration numbers set better than this? That is the beauty of the large statistics set displayed rather than a single cycle, and perhaps of the plotting against energy rather than time. The obvious systematic disagreement was not noticed in single cycle data. And it is already clear that the jumping - far beyond predicted statistical error - is present in both sets. 
Now we try the auto-fitting starting from the usual "book" values. We have not well studied the sensitivity if the fitting to the initial conditions but here we notice that if we start with the B0 parameter - the initial field for the gauss clock - lower by 1 gauss $(844 \mathrm{~g})$ then the fit selects a solution with Bdd close to zero, and the fitting reduces chisq/d very little (from what results with "book" coefficients). With B0 starting at 845g the fit goes to about the same much lower chisq/d solution for the two sets as one would expect. The JQon data/fit is shown in figure 13a. (19.475, 128453.46, 845.05, -9.05). chis/d 0.61. Following the same drill on the JQoff data gives figure 13b. and calibrations $(19.475,128453.47,845.1,-9.04)$ chis/d 0.78 . And there is nothing further to be done as far as the manual fitting strategy is concerned. The points show little curvature and the slope of the line is zero.

Comparing the calibration numbers to the plotted values above for 2013 shows that the R0 is larger here (in 2012) - by maybe .03mm and B0 is lower - by half a gauss. $\mathrm{GCcal}$ is close to the late in run value during 2013. The Bdd number is at the low end of the values found in 2013. R0 is allowed to change over months and runs- if the offsets in the AGS Orbit Display program are changed or if different sets of pickups are used. B0 is sort of the injection field and also could change a bit - though we want to be coming in at Ggamma 4.5.

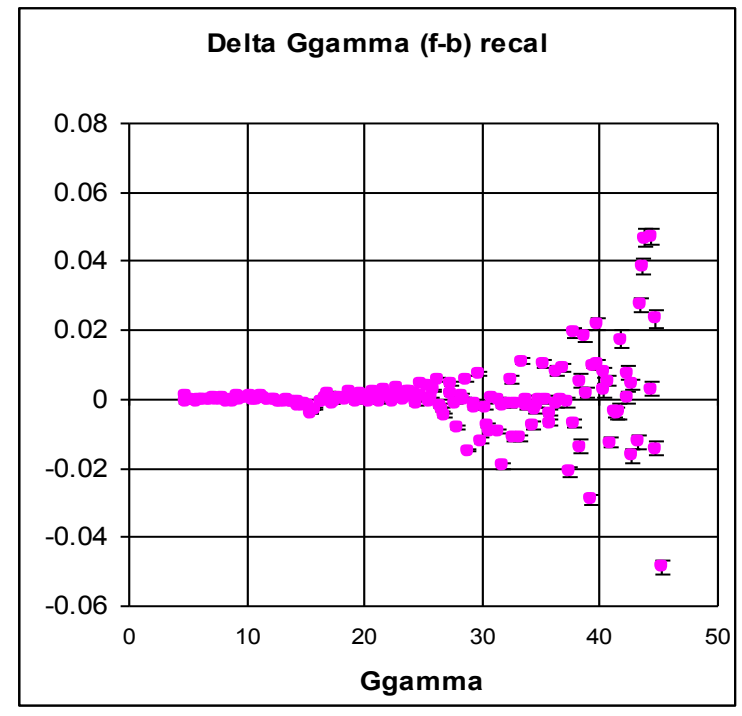

Figure 13a: autoFitting JQon

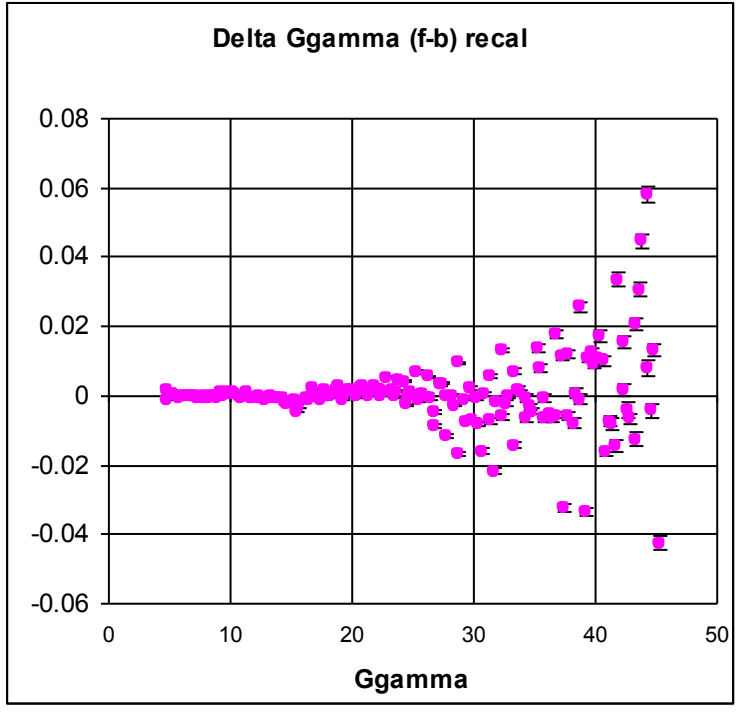

Figure 13b: autoFitting JQ off

The GCcal derived is lower that what was used for the timing by 0.015 (19.475 vs 19.49). $\mathrm{B} 0$ is also a bit shifted. The resulting change in GgammaBr is shown in figure 14. This would put the beam right on the edge of the correcting region - half in and half out all else equal. 


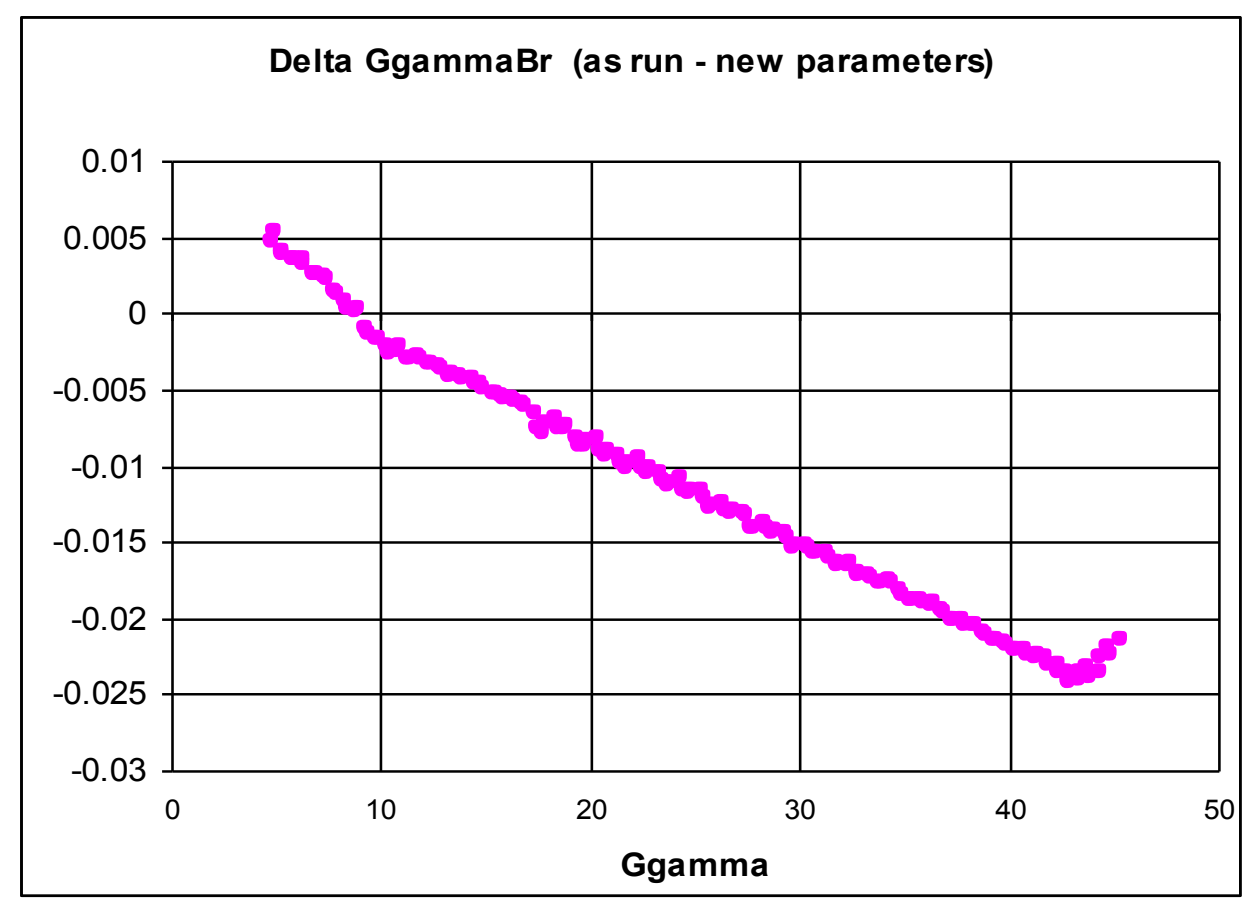

Figure 14: Predicted change in GgammaBr used in the (bad) timing creation of 25Mar12

The other thing we want from these sets is a comparison of the jumping behavior late in the cycle. Is the pattern affected by the JQs being active? This - the average differences in the two JQ situations - is shown in figure 15. The two patterns are similar. The JQoff set is not quieter. The timing set has points $1 \mathrm{~ms}$ apart and points $\sim 10 \mathrm{~ms}$ apart. The tune shifts occur in time near the pair close together. Any JQon/JQoff structure difference is not compelling.

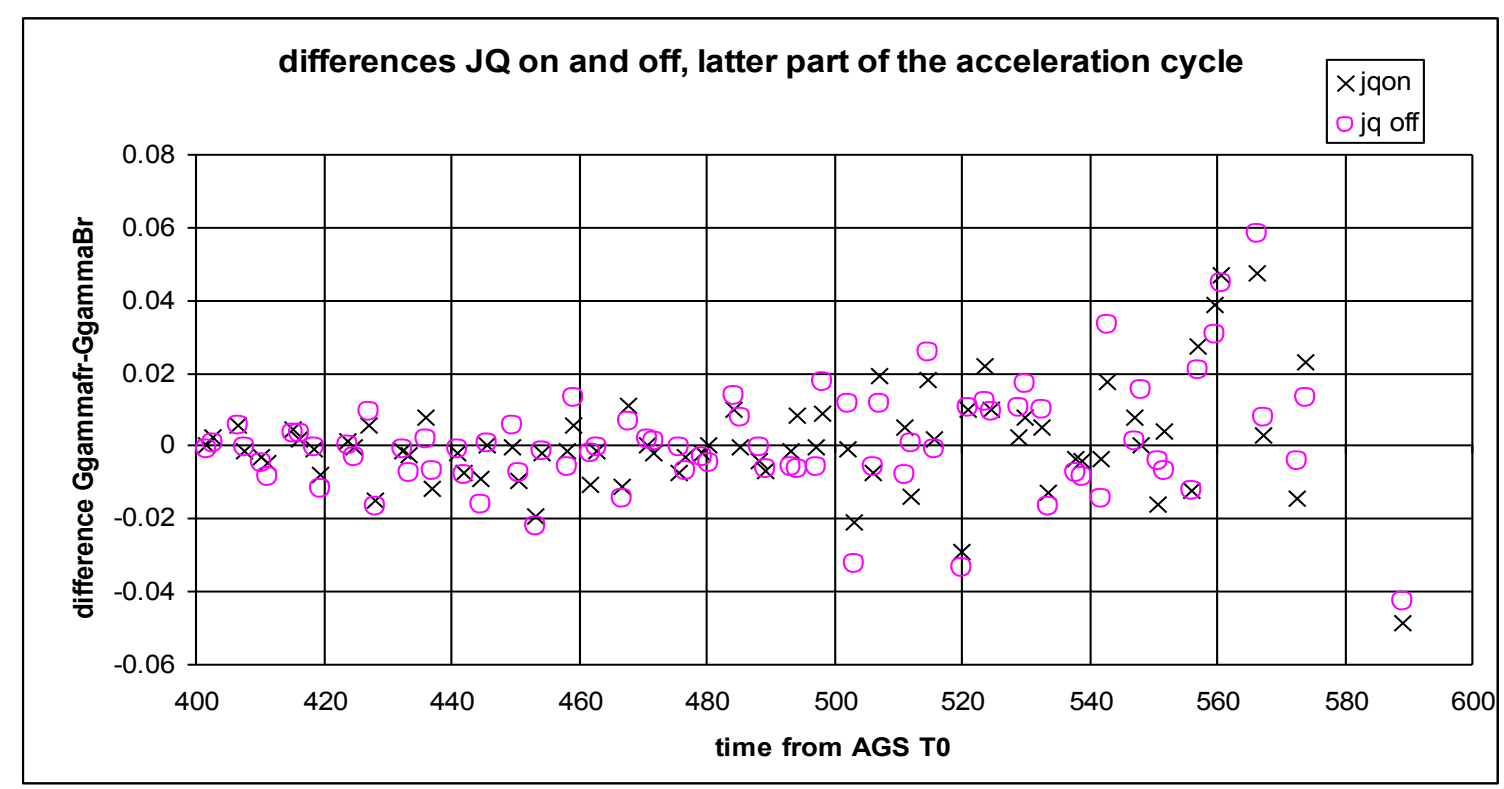

Figure 13: Usual predicted Differences (GgammaFr - GgammaBr) with JQs on and off. The jumping remains. 


\section{A List of Action Items}

The Ggamma Meter calibrations must be revisited whenever a new Jump Quad timing set is to be generated.

Revisiting the calibrations requires accumulating large data sets -200 cycles. Adding the machinery to allow getting such sets easily is necessary.

Modeling B (GCC) is not complete. The correction to the incoming count rate by adding a modulation proportional to $\mathrm{d} / \mathrm{dt}(\mathrm{dB} / \mathrm{dt})$ is not well founded. If this adjustment is wrong the whole approach is undermined. We use GgammaBr for the Jump Quad timing determination.

The jumpy behavior of the difference between GgammaBr and GgammaFr late in the acceleration cycle is not due to statistics nor to the Jump Quads and is not understood. The likely cause is associated with GgammaFr and path length changes in the machine though the discussion goes no further. A simple check of the frequency is possible if the rf group makes available an averaged version of the frequency sent to the acceleration cavities. Plugging this alternative frequency measurement into the Ggamma Meter would immediately simplify this discussion though it might well not get rid of the jumping.

The AGS gauss clock starts counting, up counts minus down counts, at AGS T0. The net accumulated counts when the beam is injected depend on both up and down calibrations and the associated calibration parameter required by the GgammaBr (B0) is not rigorously the field at injection. If the gauss counts used by the Ggamma meter started at injection, the B0 parameter becomes a real physical quantity well defined just by the measured frequency at injection and worries about the calibration of the down counts goes away.

The stability of the gauss clock - the GCcal parameter - apparently changes over weeks of time. Some investigation into this - e.g. the temperature stability of the environments of the system components - would be reasonable. Making a new gauss clock - or even tearing into this one - is probably not a good path to follow.

The real constants $(\pi, \mathrm{c}, \ldots)$ in the Ggamma Meter should be checked to be sure they are correct.

In the Ggamma Meter pet the option to plot the difference (GgammaFr - GgammaBr) against Ggamma rather than against time should be provided. 


\section{Appendix 1: Error Analysis and the Ggamma measurements at Low Energies}

Using one set of 349 cycles of data (23May13 starting 10am) the standard deviations of the measured quantities are studied. The measurements of the inputs (frequency, radius, gauss clock counts) are analyzed to yield their standard deviations over the set. The standard deviations of the reported GgammaBr and GgammaFr from the Ggamma meter are also calculated. The standard deviations of the means of these quantities are just these numbers divided by the square root of the number of measurements, here (square root 349 ) or $\sim 18$. Taking partial derivatives in the formulas in play, the GgammaBr and GgammaFr standard deviations can then also be calculated from the associated input standard deviations. Finding the standard deviations of the Ggammas both ways is just a check that the error propagation is being done properly and that using averages of the inputs is ok to calculate the average of the outputs. Figure A1 given the results.

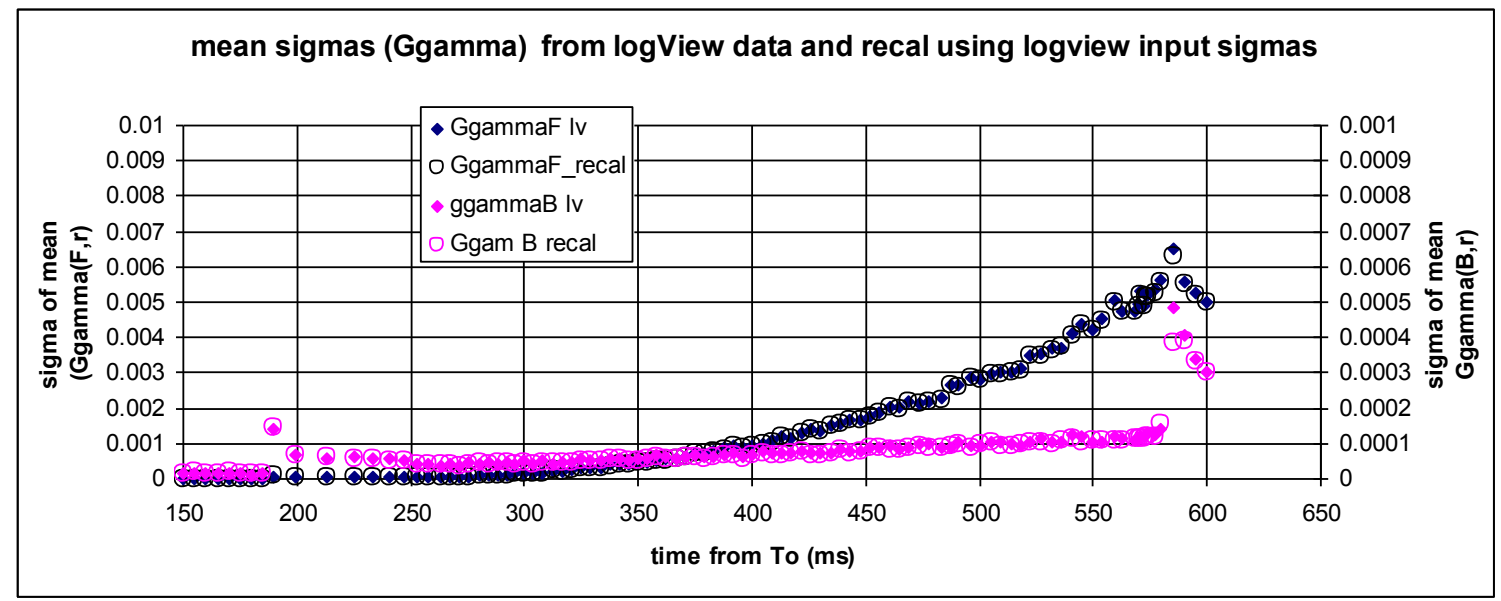

Figure A1: Reported (filled in diamonds) and recalculated (open circles) standard deviations of Ggamma

The results from $(\mathrm{F}, \mathrm{r})$ are associated with the left hand vertical scale; those from $(B, r)$ on the right. Note that the $(F, r)$ results require an order of magnitude larger scale to fit on the plot late in the cycle. As the beam becomes highly relativistic, the sensitivity to frequency becomes high. Early in the AGS cycle the situation is very different. GgammaFr has the much smaller statistical errors. A replot of figure A1 showing this region is given in figure $\mathrm{A} 2$. 


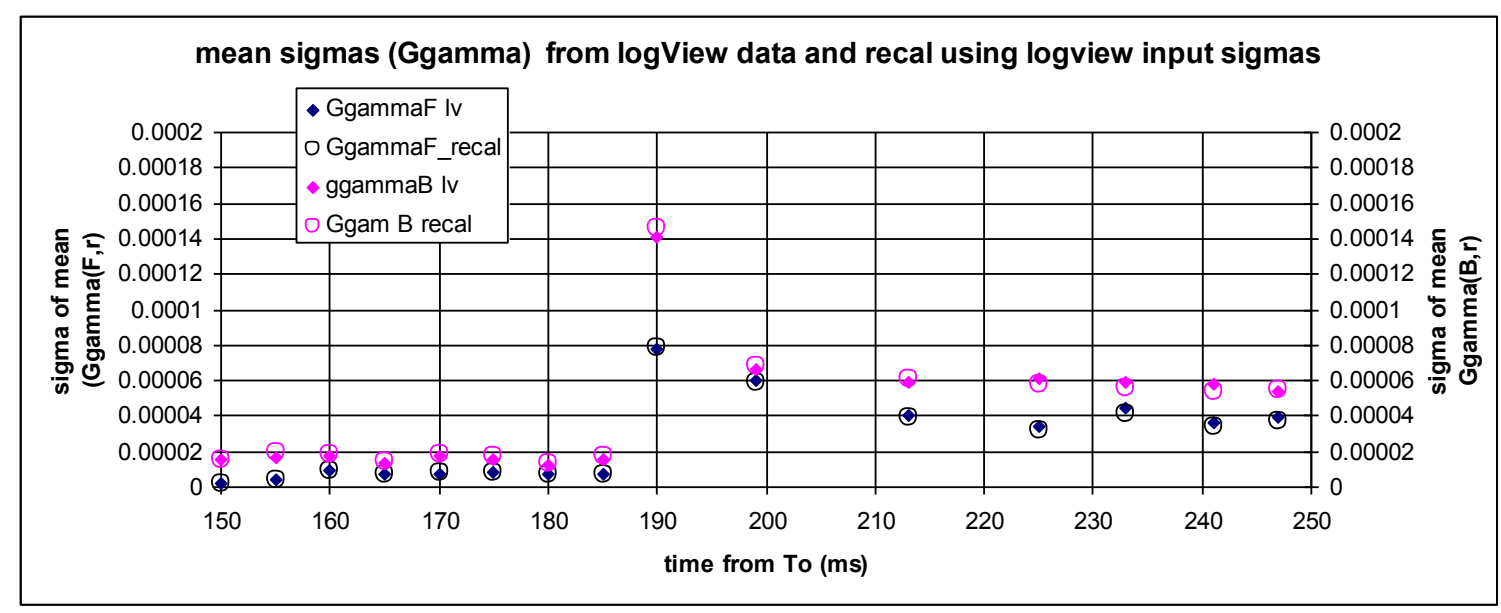

Figure A2: Replot the data in A1 for early times in the cycle and now with all data using the same vertical scale

Both sets have tiny errors early (relative to our figure of merit needs $<0.02$ ). For the gauss clock this is not particularly interesting as a determination of Ggamma since counting only starts at injection - at $150 \mathrm{~ms}$. The reported GgammaBr depends mostly on the B0 parameter entered into the machinery. For GgammaFr the tiny errors indicates the relatively weak dependence on errors in both frequency and radius and hence the absolute strength of the result. The step at 190ms correlates (I think) with the shift from "flat top" bank to the "pulsed" bank of the main magnet power supply.

These standard deviations can be broken out into the piece from each of the inputs (i.e. frequency, gauss clock counts and radius $r$ ) and that is given in figure A3.

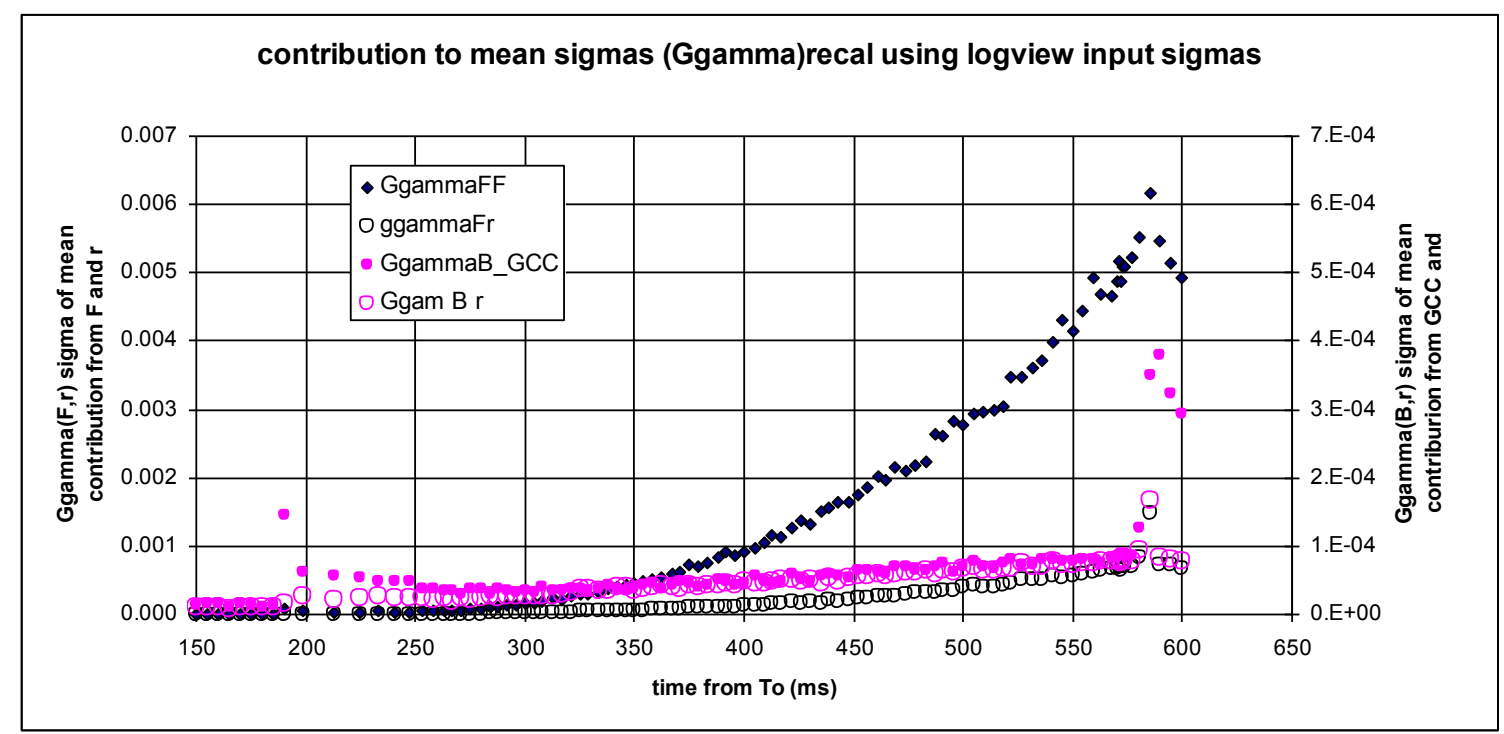

Figure A3: Contributions to the Ggamma standard deviations from each input 
The black points are associated with GgammaFr and are plotted on the left scale, again an order of magnitude coarser than the right GgammaBr scale. That the frequency error dominates at higher energy is clear and expected. Reducing this variation motivates the desire for wider frequency windows and larger data sets.

This is not an issue early in the cycle. Again the (frequency and radius) data from early in the cycle in figure A3 are replotted in figure A4, now only near injection. And the measured standard deviations of the means of all three variables are given in figure A5. The variation in the reported GgammaFr due to measurement fluctuations - in F and $\mathrm{r}-$ do not change the answer at anywhere near the resolution of interest i.e. $<.02$.

The measurement standard deviations are tiny relative to a problem level. We want to extract a stronger conclusion from this data set. The standard deviations in the (F and r) mean measurements at the first time in the cycle (from figure A5) are ( $0.4 \mathrm{~Hz}$ and $0.005 \mathrm{~mm})$. From figure A4 the Ggamma errors that result are (1e-6 and 4e-6). How much can $\mathrm{dF}$ or $\mathrm{dr}$ be increased before the effect on Ggamma exceeds say 0.001 -(well below our 0.02 standard)? dF could be increased by $\times 1000$ or to $400 \mathrm{~Hz}$ and $\mathrm{r}$ could be increased by $\mathrm{x} 250$ or to $1.25 \mathrm{~mm}$.

To know GgammaFr absolutely requires knowing (Ro +r) and F absolutely. Our well known " $r$ " gets added to the parameter Ro which is not so well known. Nevertheless late in the cycle Ro's value cannot change by much beyond $0.2 \mathrm{~mm}$ without pulling the difference between GgammaFr and GgammaBr "off the page". Provided we can assume that Ro is the same throughout the cycle, its uncertainty is of order $0.2 \mathrm{~mm}$ and we are a factor of five below where GgammaFr at injection is beginning to be affected. It is this argument - getting Ro from high energy measurements and applying it to injection measurements that allows the injection energy and hence the starting field for GgammaBr to be well known.

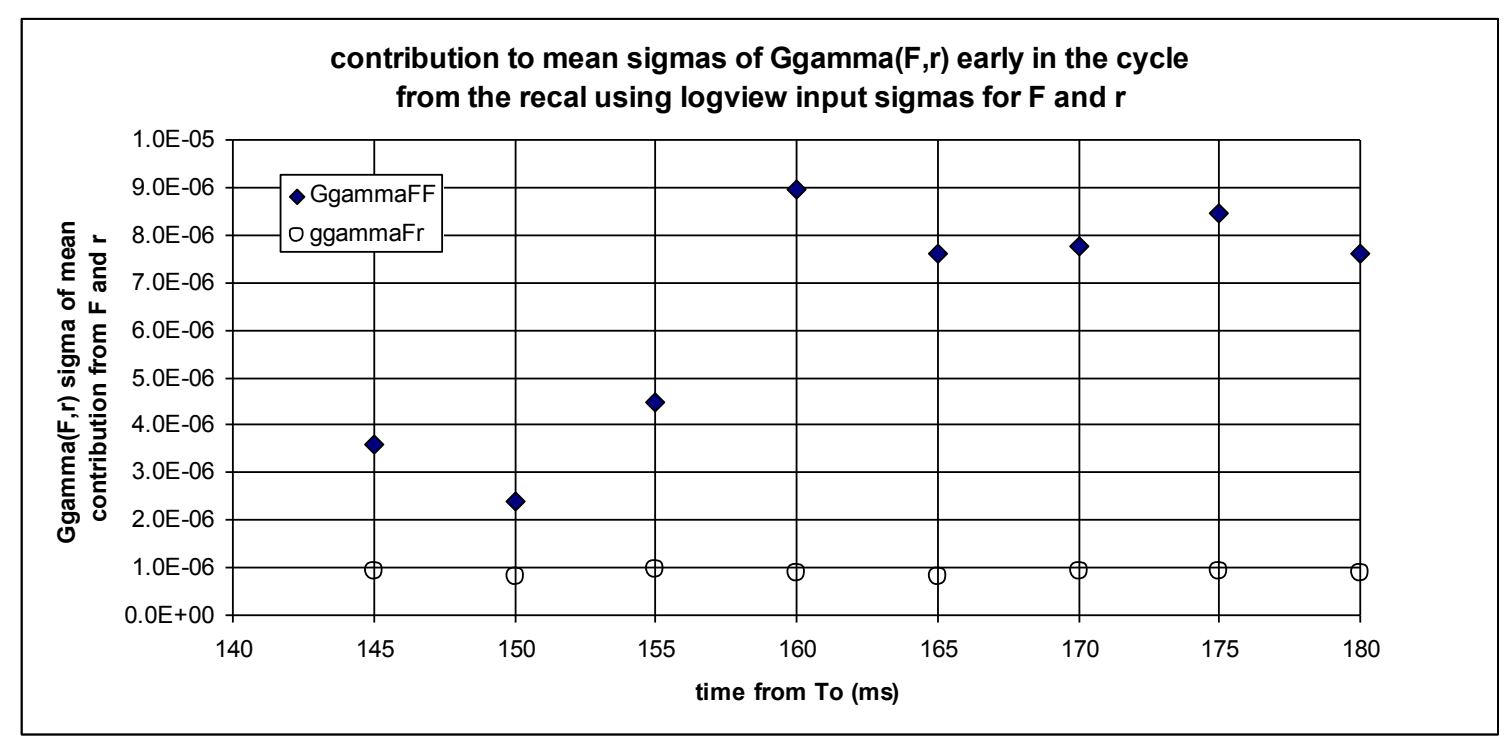

Figure A4: Here the same data - from frequency and radius is replotted with expanded scales. 


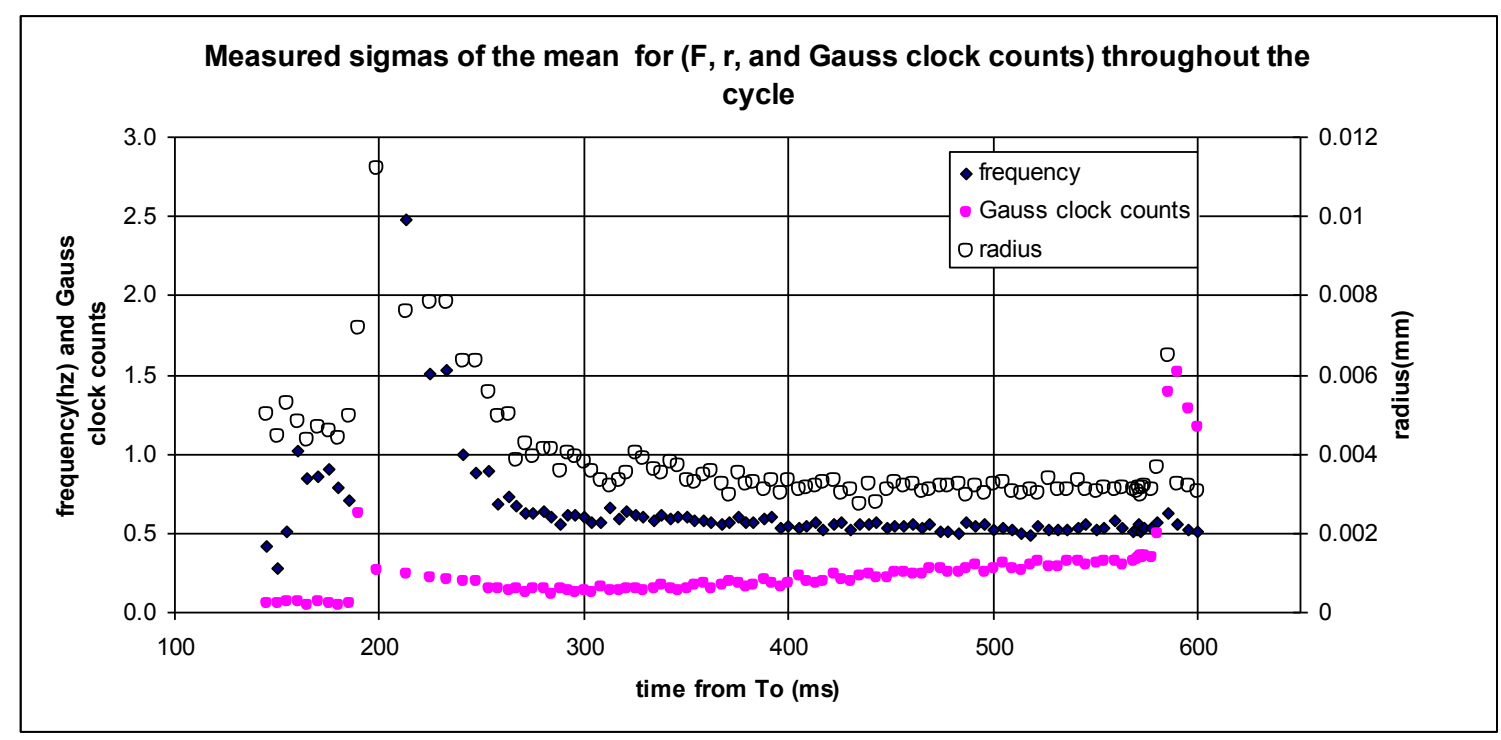

Figure A5: the measured standard deviations of the mean in F, r, and GCC) over our 249 cycle set

\section{Appendix 2: Correcting the measured frequency for $\mathbf{d}^{2} \mathbf{F} / \mathrm{dt}^{2}$}

We measure the beam frequency (rf accelerating cavities frequency really) over a window in time. The accuracy of the reported frequency increases as this window grows, but the answer is the average frequency over the window. If the frequency is fixed or changing linearly this is just fine. We shift the window to be centered on our "time of interest". If the frequency has a piece with a quadratic time dependence we still get the average frequency but not the value at the center of the window. We can correct for this effect, but how big is it?

We ask this question of the "model" - a lazy man's way to learn. The following figure gives the result. Remember that the model has a perfectly linear $\mathrm{B}(\mathrm{t})$ and so unphysical changes at the start and end. From this we conclude that a correction seems unnecessary throughout the cycle for windows less than $5 \mathrm{~ms}$ wide. We could include a correction for frequency width in the machinery and check that it doesn't change the reported GgammaFr. In order to do this we need to figure out $\mathrm{d}^{2} \mathrm{~F} / \mathrm{dt}^{2}$ in a robust way certainly not from the frequency measurement. It could be calculated from the main magnet function or from the measured gauss clock numbers. 


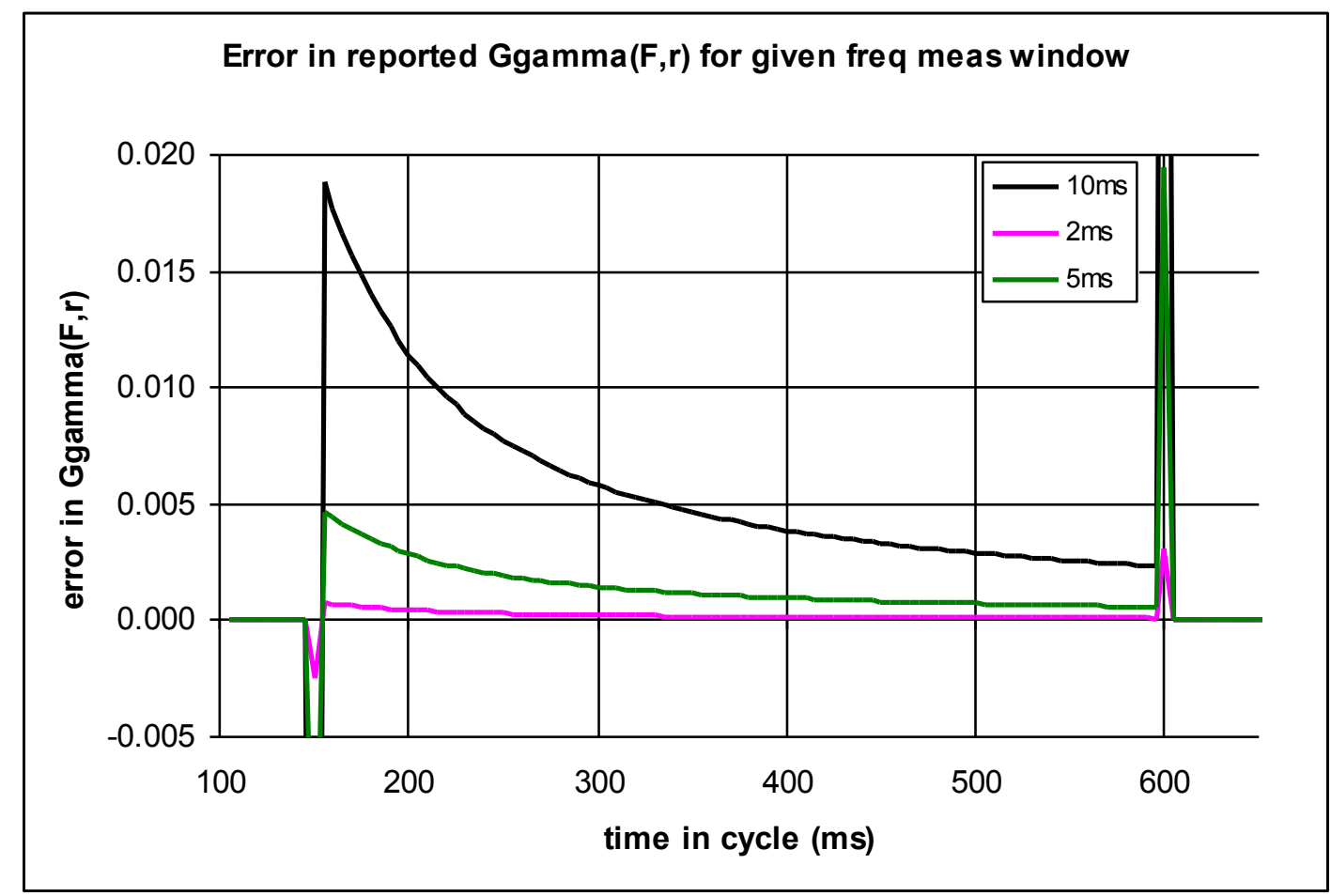

Figure29: Error introduced due to not correcting for the frequency window and $\mathrm{F}(\mathrm{t})$ nonlinear dependence. Even a $5 \mathrm{~ms}$ window (which we rarely can use) is not a big problem. 
VII. Power Point Presentation at Haixin's Polarization meeting 12Feb14

(just the slides from the 12 feb talk and added here as a convenience. This should be a shorter version of the above note.)

\section{Calibrating the AGS Gauss Clock mostly the old way}

leif ahrens 12Feb14

\section{AGS Ggamma Meter}

- GgammaBr(t) and GgammFr(t) are outputs from the AGS "Ggamma meter" machinery (Pet)

- Inputs to the meter are :measurements [GCC(t), F(t) r(t)] and calibrations [ RO, B0, Gccal, Bdd ]

- The machinery calculates the beam energy (or Ggamma) based on either

GgammaBr(t) <= \{GCC(t),r(t), d/dt(dB/dt)(t)[GCcal, Bo, Bdd]\}

or

GgammaFr $(t)<=\{F(t), r(t))[R 0]\}$ 


\section{The Calibration Exercise}

- so the Ggamma meter provides GgammaBr(t) and GgammaFr(t) using whatever calibration numbers it is given.

- We postulate that if $\mathrm{GgammaBr}(\mathrm{t})=\mathrm{GgammaFr}(\mathrm{t})$ then that $\mathrm{GgammaBr}(\mathrm{t})$ is close enough to the true Ggamma(t)) to allow adequately accurate resonance crossing times to be calculated (back on slide 2)

- So the game is to figure out how to make the two energy determinations equal which means minimizing the difference between them throughout the AGS acceleration cycle

- We try to gain some insight from a simple model

\section{A Simple Model for Measuring Calculated Ggamma sensitivities}

We assume that the magnet cycle in the AGS is given by a linear ramp. The parameters are chosen to be close to typical proton cycles. The transitions from flats to ramping are not at all realistic having discontinuous slopes in the model.

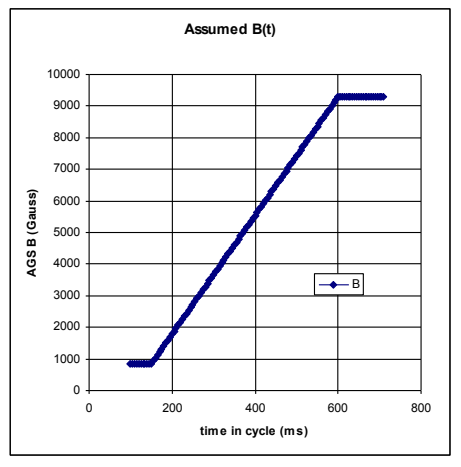

$B(t)$, rho $=>$ gamma $(t)$, beta $(t)$,

Ggamma(t) (on the central orbit - rho)

$($ beta $(t), R o)=>$ frequency $F(t)$

$\mathrm{B}(\mathrm{t}), \mathrm{Gccal}=>\mathrm{GCC}(\mathrm{t})$

So we have the perfect set.

Then we can introduce "errors" into

the pathlength (through Ro) messing up

GgammaFr(t)

Or into Gccal messing up GgammaBr 
So that is what is shown here. The effect of changing Ro $(128453 \mathrm{~mm})$ by $0.2 \mathrm{~mm}$ (magenta) or of changing GCcal ( $19.5 \mathrm{GCC} /$ gauss) by 0.02 (black) gives errors in Ggamma that grow to 0.04 at Ggamma $=45$. Our figure of merit tolerance ishalf this - 0.02 in Ggamma.

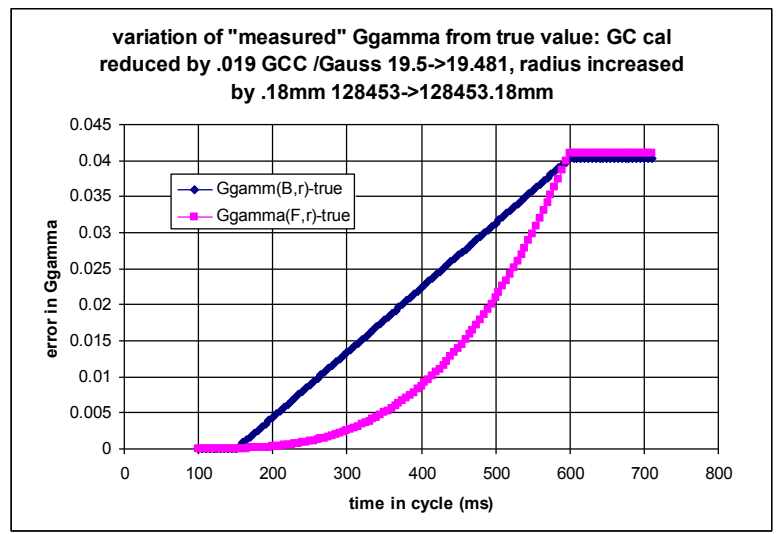

If GgammaBr is wrong by .02 we are in trouble.

The corresponding error in Gccal is 0.01 (GCC/gauss)

And the corresponding error in $R o$ is $0.1 \mathrm{~mm}$

Let's look at some data. 23may13 231 cycles

This shows the difference between GgammaFr and GgammaBr as the machine was running 23May13 and using the calibration set that was "active" in the Ggamma meter.

The horizontal scale for the left plot is Ggamma for the right is time in cycle. The vertical is the difference reported between GgammaFr and GgammaBr. Measurements well out on the flattop are evident there. Our concern level is 0.02 which is crossed at Ggamma 20.

But notice other properties of this data set - which are very typical: There is huge scatter in points at energies above Ggamma 20 which are not explained by the cycle-to-cycle scatter (error bars show the error of the mean over the set).
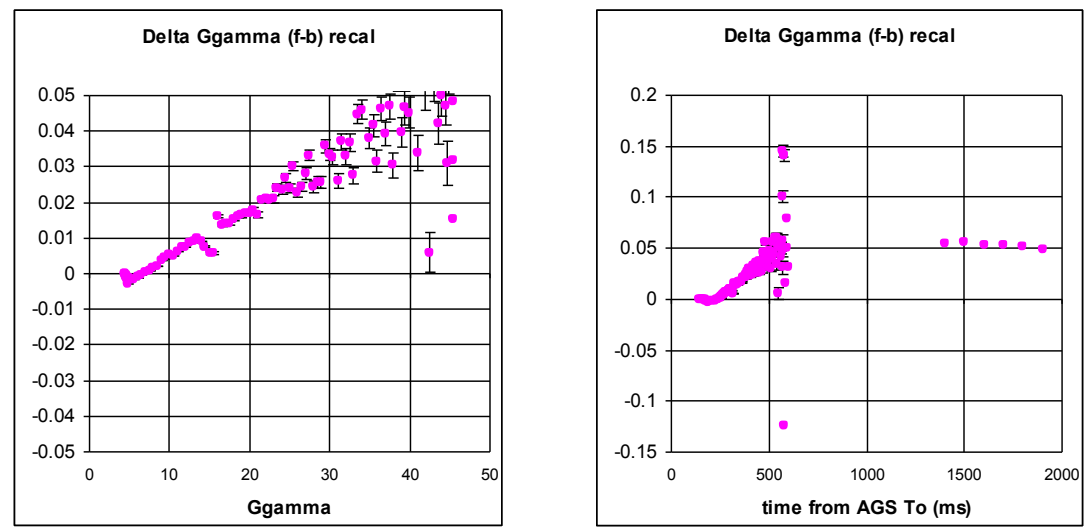
Now "play" with this data set a bit - what does it look like with other cal sets?

First put in what I call "book" parameters - numbers close to the designs and no dependence of the GgammaBr on d/dt(dB/dt) Gccal, Ro, Bo, Bdd $19.50,128453.0,846,0$

The real problem here is that the difference stays within our 0.02 criterion - which might cause us to think Gccal was ok.

Next try fitting this set to minimize the differences. The result has structure curvature - that our model would associate with an incorrect Ro. But it has

much much more - early structure beyond our model's ability, large real scatter later in acceleration (again not understood) and large error bars late due to

frequency scatter the sensitivity to frequency (effectively allowing the fit to disregard these points). $19.52,128452.68,845.8,0$
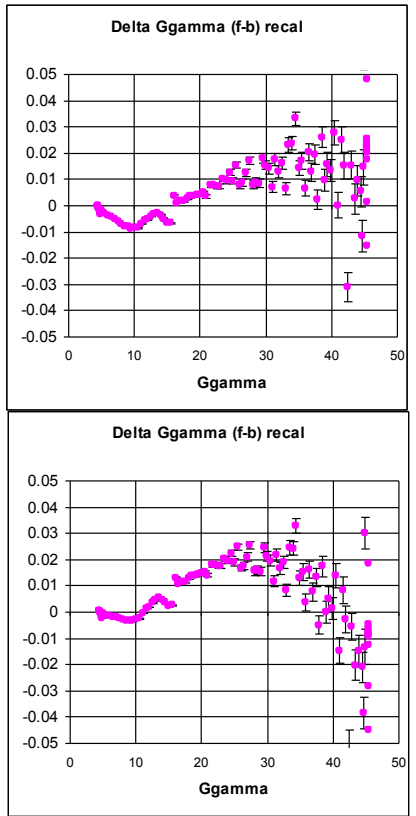

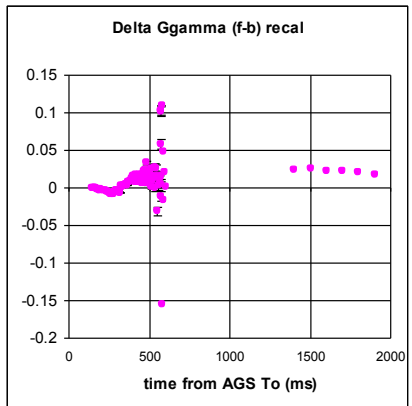

Delta Ggamma (f-b) recal

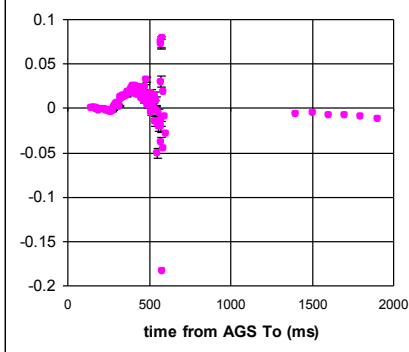

Gccal went from 49.5 to 49.52 - a huge change. Continuing in this exercise, we remove the flattop points from the fitting and try again. This gives the plots on the right. The shape is greatly improved though any curvature is obscured by the point scatter. The differences on flattop are systematically away from zero.

And the Gccal dropped from 19.5 to 19.474. Again a huge change by our rules.

$19.474 \quad 128453.07 \quad 845.8-10.4$

And just for completeness, we give a solution 1) fixing $B 0$ to give a zero difference at injection and then removing curvature shifting Ro and flattening using Gccal, all of thie "by eye".

$\begin{array}{llll}19.467 & 128453.25 & 846 & -11\end{array}$

The last two solutions are nearly close enough give some comfort. And for this set the flattop points have come back to near zero.
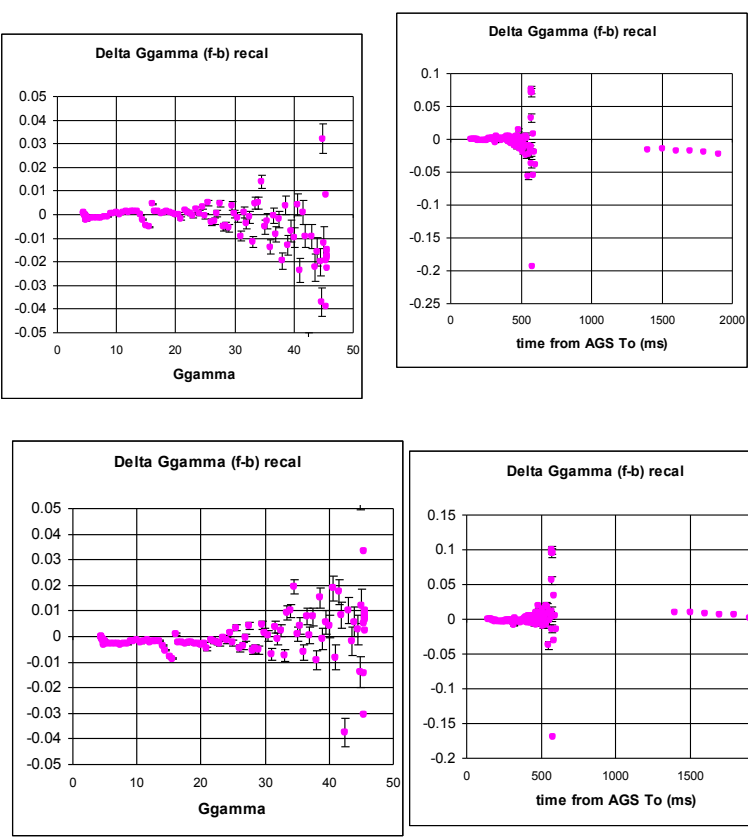

Delta Ggamma (f-b) recal

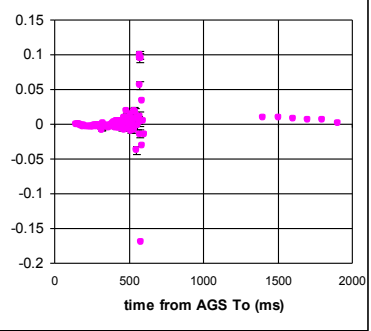


The "bottom line" from this exercise as far as the gauss clock calibration is concerned: the GCcal coefficient steps from

19.5

book

19.52

(full fitting)

19.474

(exclude flattop and full fit)

19.467

"by hand" - (Bo from GgammaFr, remove curvature by eye)

19.475

(Yann_29May

A variation in this slope parameter of 0.01 moves the reported Ggamma by 0.02 or the times by 200 usec. For our "fittings" we have a spread of 0.05 (500usec), the three that exclude flattop vary by 0.007 (70usec).

We should say in passing that this is a well behaved data set. Similar processing on other sets frequently does not look good and does not fit on the flattop.

\section{Problems:}

The early difference though tiny has smooth structure which is beyond our model - even including the Bdd term. Is this a problem with the GgammaFr (the radius) or with GgammaBr?

The late acc cycle differences have structure that is enormous relative to the mean standard deviation. The worst is somehow associated with $36+$ but the other stuff is also problematic. If we could fix this we would either gain more confidence in the fitting method or see more clearly that it is wrong.
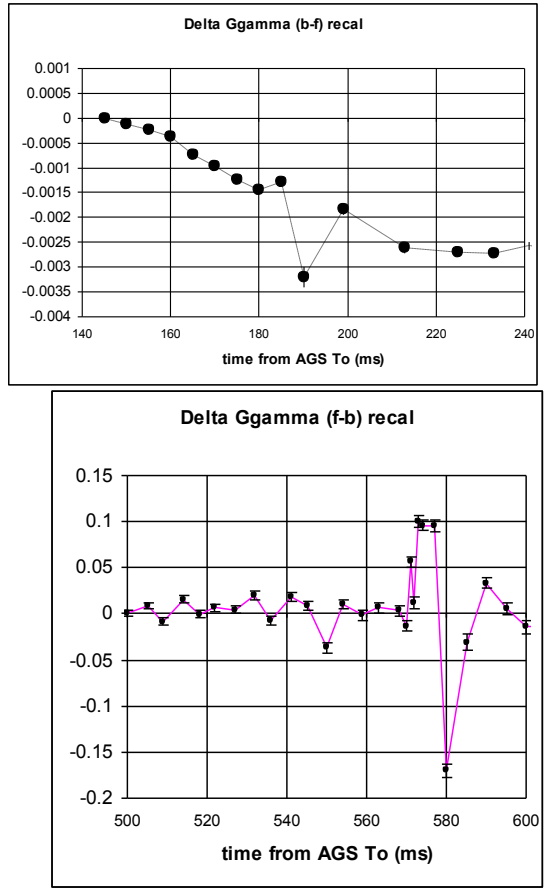
Nevertheless: life being short, we go with the fitting, ignore flattop and plot GCcal measured vs time over Run13

The black points are what is just described. The magenta are "eye" fits (also ignoring the flattop points). The green bar is our usual figure-of-merit error - the shift that would result in a 200 usec timing shift if a JQ timing run were taken with the active calibration number this far from the truth.

This looks almost plausible - better than we deserve - except for the fit points at 28May. Can the gauss clock

calibration really jump around on such a short

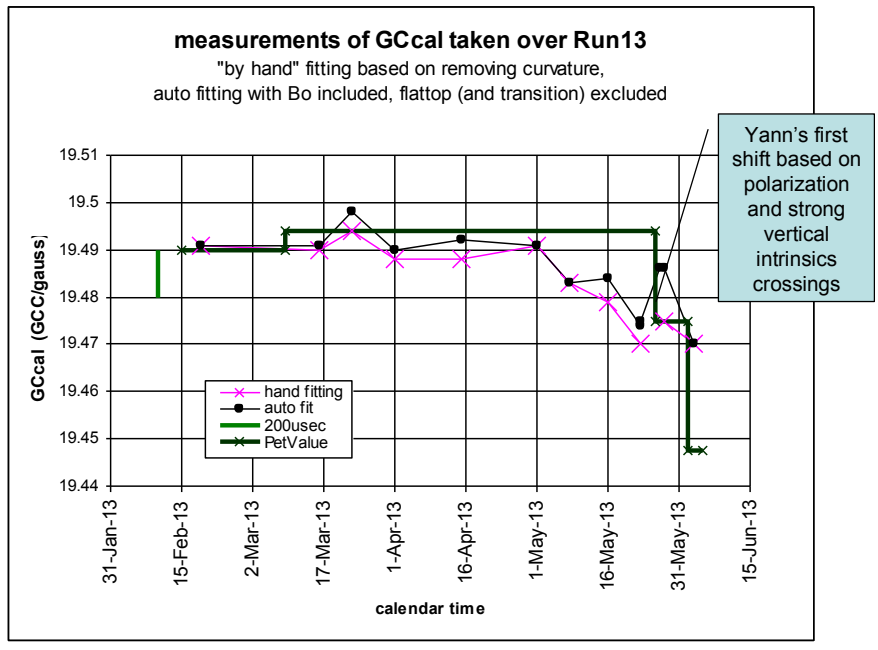
time scale?

Maybe this is too hard i.e. learning the calibration factors from the differences (GgammaBr - GgammaFr). Yann's learning Ggamma at many points during the cycle is a somewhat alternative path. It provides a few or a large set of additional constraints which could be included in the fitting. Even one time point late in the cycle but before flattop would be very useful.

But let's continue and see what we need:

Apparently the calibrations have to be checked before data is taken to create a new Jump Quad timing file.

Accumulation and analysis of large data sets needs to be much easier to make the calibration check doable.

The model - B(GCC) vs time or some radius effect that looks like a GCC error - is not understood. The ad hoc Bdd correction makes the "eye" fitting possible, but unexplained difference behavior remains. Since we use GgammaBr for the timing, this is very important. Maybe the Bdd guess is wrong. If somehow GgammaFr is responsible for the early structure then this whole construct and the derived GCcal is wrong. 
The differences go jumping around late. Maybe this is demonstrating a problem with the frequency meter. We may have an option here, namely the rf group's knowledge of the frequency sent to the cavity. To use that info it needs to be averaged starting from raw very time dense numbers. The Ggamma meter is ready to accept such a table.

The Gauss clock counts used in the machinery start counting at AGS TO. That means "Up and Down" counts are added into the sum across the sometimes long injection porch. This adds some confusion and means the B0 in the fitting is rigorously neither the field at injection nor at T0. To keep this parameter's interpretation simple we should figure out how to start counting the gauss clock counts used in the Ggamma meter at - or just before - injection.

The stability of the gauss clock is questioned. Some investigation into this - e.g. the temperature stability of the environment for the electronics? - is reasonable. Making a new gauss clock - or even climbing into this one - are probably not appropriate options. 